\title{
Atmospheric resonances and their coupling to vibrations of the ground and waves in the ocean
}

\author{
Oleg A. Godin ${ }^{*}$ (D), Nikolay A. Zabotin ${ }^{2}$ and Liudmila Zabotina ${ }^{2}$
}

\begin{abstract}
Observations of the ionosphere with the airglow, GPS-TEC, and HF radar techniques reveal a resonant response of the middle and upper atmosphere to broad-band excitation by earthquakes, volcano eruptions, and convective storms. The resonances occur at such frequencies that an atmospheric wave, which is radiated at the ground level and is reflected from a turning point in the middle or upper atmosphere, upon return to the ground level satisfies boundary conditions on the ground. Using asymptotic and numerical models of atmospheric waves, this paper investigates atmospheric resonances and their excitation by seismic waves and infragravity waves in the ocean. It is found that "buoyancy" resonances with periods up to several hours arise in addition to "acoustic" resonances with periods of about 3-5 min. The acoustic and buoyancy resonances occur, respectively, on the acoustic and gravity branches of the dispersion curve of acoustic-gravity waves. Buoyancy of the atmosphere is important for the resonances of both kinds. Acoustic resonances are found to be sensitive to the temperature profile, especially around mesopause and tropopause, and are predicted to be a seasonal phenomenon in polar atmosphere. Unlike acoustic resonances, buoyancy resonances exhibit high sensitivity to the wind velocity profile and its variations. The resonances correspond to most efficient coupling between the atmosphere and its lower boundary and are promising for detection of such coupling.
\end{abstract}

Keywords: Acoustic-gravity waves, Infragravity waves, Air-sea interaction, lonosphere, Normal modes

\section{Introduction}

Atmosphere is known to respond in a resonant way to broad-band excitation associated with earthquakes (Shinagawa et al. 2007; Choosakul et al. 2009; Saito et al. 2011; Rolland et al. 2011; Ogawa et al. 2012; Cahyadi and Heki 2014; Jin 2018), volcanic eruptions (Widmer and Zürn 1992; Kanamori et al. 1994; Tahira 1995; Zürn and Widmer 1996; Watada and Kanamori 2010; Aoyama et al. 2016; Nakashima et al. 2016), turbulence in mountain regions (Bedard 1978), and severe meteorological events such as convective storms (Chimonas and Peltier 1974; Jones and Georges 1976; Pilger et al. 2013) and tornados

\footnotetext{
*Correspondence: oagodin@nps.edu

${ }^{1}$ Department of Physics, Naval Postgraduate School, Monterey, CA 93943, USA

Full list of author information is available at the end of the article
}

(Nishioka et al. 2013). The resonances are manifested as narrow frequency bands of greatly amplified oscillations and have periods of 3-5 min. Although the resonances are referred to as acoustic resonances, buoyancy effects are not negligible at such frequencies (Gossard and Hooke 1975; Jones and Georges 1976; Godin 2015). In addition to transient responses of the atmosphere to an impulsive excitation, acoustic resonances are prominent in long-term averages of the seismic noise spectrum (Nishida 2014). Theoretical considerations and observational evidence indicate that acoustic resonances are also present in the solar atmosphere (Taroyan and Erdélyi 2008; Yuan et al. 2016). Simultaneous post-earthquake observations of sharp peaks in the frequency dependence of barometric signals on the ground and magnetic signals 
due to field-aligned currents in the ionosphere (Iyemori et al. 2013) provide a direct proof that on Earth the atmospheric oscillations in acoustic resonances extend from the ground to the upper atmosphere. Most of the available experimental data on acoustic resonances originates from ionosphere sounding with ground-based HF radars (Jones and Georges 1976; Bedard 1978; Tahira 1995; Ogawa et al. 2012), measurements of variations of the slant total electron content (TEC) using signals from GPS satellites (Choosakul et al. 2009; Rolland et al. 2011; Saito et al. 2011; Ogawa et al. 2012; Nishioka et al. 2013; Cahyadi and Heki 2014; Aoyama et al. 2016; Nakashima et al. 2016; Reddy et al. 2016; Jin 2018; Liu and Jin 2019), and measurements of nocturnal airglow emissions (Pilger et al. 2013). All these measurements rely on the physical processes in the upper and middle atmosphere.

Strong excitation of atmospheric oscillations in the 1-3 $\mathrm{mHz}$ band, which lies below the frequencies of acoustic resonances, was observed (Rolland et al. 2011) over Pacific off Japan after the 2011 Tohoku-Oki earthquake (Ozawa et al. 2011) and associated with the earthquake-generated tsunami. Spectrum of the observed atmospheric oscillations lies above the frequency band, where most of the tsunami energy was concentrated (Heidarzadeh and Satake 2013). We believe that the atmospheric oscillations in the $1-3 \mathrm{mHz}$ band observed by Rolland et al. (2011) are an expression of a resonance or resonances of the atmosphere with frequencies lower than those of the acoustic resonances. Another possible manifestation of low-frequency atmospheric resonances was found (Zabotin et al. 2016) in long-term averages of background wave activity in the thermosphere over the US East Coast.

Analytic and semi-analytic approaches (Jones and Georges 1976; Tahira 1995; Matsumura et al. 2009) to prediction of acoustic resonances often assume vertical propagation of AGWs in the atmosphere and relate occurrence of the resonances to waves reaching an altitude, where their frequency equals the acoustic cutoff frequency (Gossard and Hooke 1975). Any upward propagating atmospheric wave with a finite speed of propagation along the ground surface reaches a turning point, where it travels horizontally, before reaching the cutoff (Hines 1965; Godin 2015). This makes the assumption of vertical propagation questionable. Atmospheric resonances also emerge in numerical models of the wave field generated by a compact source (Matsumura et al. 2011, 2012), particularly in the models that are based on summation of normal modes of the coupled solid Earthocean-atmosphere system (Lognonné et al. 1998; Artru et al. 2001, 2004; Kobayashi 2007; Watada and Kanamori 2010; Rolland et al. 2011). The numerical models, while very powerful in many respects, disregard the effect of the background wind on waves in the atmosphere and are not well suited to study and provide physical insights into the influence of geographic and temporal variations of the temperature profile and winds in the atmosphere on the properties of its resonances.

In this paper, we investigate resonant oscillations of the atmosphere from the viewpoint of their excitation by extended sources in the solid ground, ocean, or its ice cover. The sources are modeled as vibrations of the lower boundary of the atmosphere. We apply the consistent WentzelKramers-Brillouin (WKB) approximation (Godin 2015) to study atmospheric resonances, their geographic and temporal variability, and the atmospheric properties that lead to occurrence of the resonances. The asymptotic theory is supplemented by direct numerical solution of the wave equation when necessary. Using asymptotic and numerical models of atmospheric waves, we quantify the effects of wind velocity and temperature profiles on the properties of the atmospheric resonances and relate the amplitudes of ground-level excitations and upper atmosphere manifestations of the resonances. The analysis is not limited to acoustic resonances but extends to lower frequency resonances with periods as long as several hours.

The paper is organized as follows. In "Asymptotic modeling of atmospheric resonances", the WKB approximation for AGWs (Godin 2015) is used to quantify the conditions necessary for resonances to occur in a continuously stratified atmosphere with gradually varying temperature and wind velocity and to determine the frequency of resonant oscillations of the atmosphere excited by waves traveling along its lower boundary. Excitation of atmospheric resonances by finite sources is also considered in that section. A simple numerical model of excitation of atmospheric oscillations by ground-level vibrations is presented in "Atmospheric resonances at high and mid-latitudes". The numerical approach serves to verify the asymptotic theory and relax the assumption of gradual variation of atmospheric parameters with altitude. The numeric and asymptotic approaches are employed in subsections "Acoustic resonances" and "Buoyancy resonances" to investigate atmospheric resonances under mid-latitude and polar conditions, using Wallops Island, Virginia, and McMurdo, Antarctica, as specific examples. The choice of locations is motivated by previously reported long-term observations of wave activity in the middle and upper atmosphere at these sites (Chen et al. 2016; Chen and Chu 2017; Godin et al. 2015; Zabotin et al. 2016). In "Coupling to infragravity waves in the ocean", we show that certain atmospheric resonances can be readily excited by long surface gravity waves in the ocean and that such excitation provides a possible explanation of some of the previously reported robust features of wave activity in the middle and upper atmosphere observed at Wallops Island. "Conclusion" summarizes our findings. 


\section{Asymptotic theory of atmospheric resonances Environmental model and wave description}

Consider acoustic-gravity waves (AGWs) in an atmosphere with sound speed $c$, background flow velocity (wind) $\mathbf{u}$, and density $\rho$. The atmosphere is an ideal gas with the ratio $\gamma$ of specific heats at constant pressure and constant volume. Introduce a Cartesian coordinate system $\mathbf{r}=(x, y, z)$ with horizontal coordinates $x$ and $y$ and a vertical coordinate $z$ increasing upward. Parameters of the atmosphere are assumed to be functions of altitude $z$. The quantity

$$
h=c^{2} / \gamma g,
$$

where $g$ is the apparent acceleration due to gravity, will be referred to as the scale height. In the isothermal atmosphere, the density decreases with altitude by the factor $e$ over the distance $h$. The lower boundary of the atmosphere is located at $z=0$ and can represent either solid ground, ocean surface, or ice. Because of the large density contrast with the air, the boundary is modeled as a rigid surface.

We consider linear waves, use complex notation for the wave field, and imply the $\exp (-i \omega t)$ dependence on time $t$ in monochromatic waves. We assume that waves in the atmosphere are generated by wave processes at $z<0$. Here, the source of waves in the atmosphere is represented by vibrations of its lower boundary, with vertical displacements of the boundary having the dependence $\exp (-i \omega(t-x / V))$ on time $t$ and horizontal coordinates. For normal modes, $V$ represents their phase speed. AGW boundary conditions require that the vertical displacement of air at $z=0$ equals that of the boundary (Gossard and Hooke 1975; Godin 1997).

AGWs in the atmosphere will be modeled in the WKB approximation (Budden and Smith 1976; Godin 2015). The approximation represents a systematic asymptotic solution of equations of hydrodynamics for linear waves and should be distinguished from ad hoc approximations (Pitteway and Hines 1965; Einaudi and Hines 1970; Gossard and Hooke 1975; Jones and Georges 1976; Fritts and Alexander 2003) using the same name. Lagrangian pressure perturbations in upward- and downward-propagating waves are given by (Godin 2015)

$$
\begin{aligned}
\tilde{p}(z)= & \tilde{p}(0) \sqrt{\frac{\rho m(0)\left(\omega_{d}^{2}-g^{2} k^{2} / \omega_{d}^{2}\right)}{\rho(0) m\left[\omega_{d}^{2}(0)-g^{2} k^{2} / \omega_{d}^{2}(0)\right]}} \\
& \times \exp ( \pm i[\varphi(z)+\chi(z)]),
\end{aligned}
$$

$$
\begin{gathered}
\varphi(z)=\int_{0}^{z} m\left(z_{1}\right) \mathrm{d} z_{1}, \\
\chi(z)=\int_{0}^{z} \frac{\mathrm{d} z_{1}}{2 m}\left[\frac{g k^{2}}{\omega_{d}^{2}} \frac{\mathrm{d}}{\mathrm{d} z_{1}} \ln \left(\frac{h}{\omega_{d}^{4}-g^{2} k^{2}}\right)+\frac{1}{2 h} \frac{\mathrm{d}}{\mathrm{d} z_{1}} \ln \left(\frac{\omega_{d}^{4}-g^{2} k^{2}}{\omega_{d}^{2}}\right)\right] .
\end{gathered}
$$

Here $\phi, \chi$, and $m$ are the phase integral (also referred to as eikonal), Berry phase, and vertical component of the wave vector, $\mathbf{k}$ is the horizontal wave vector, $\omega_{d}=\omega-\mathbf{k} \cdot \mathbf{u}$ is the intrinsic wave frequency, and

$$
m^{2}=\frac{\omega_{d}^{2}}{c^{2}}-k^{2}-\frac{\gamma^{2} g^{2}}{4 c^{4}}+\frac{(\gamma-1) g^{2} k^{2}}{\omega_{d}^{2} c^{2}} .
$$

The intrinsic frequency has the meaning of wave frequency in the reference frame moving with the local wind. For definiteness, we assume that $m>0$ when $m^{2}>0$ and Im $m>0$ when $m^{2}<0$. In upward- and downwardpropagating waves, vertical displacement of fluid particles is given by

$$
w(z)=\frac{g k^{2} \omega_{d}^{-2} \pm i m-1 / 2 h}{\left(\omega_{d}^{2}-g^{2} k^{2} \omega_{d}^{-2}\right) \rho} \tilde{p}(z) .
$$

The Lagrangian pressure perturbation (i.e., the pressure perturbation due an AGW in a moving fluid parcel) and the Eulerian pressure perturbation $p$ (i.e., the waveinduced pressure change at a fixed point in space) are related by the equation $\tilde{p}=p-\rho g w$ (Godin 1997).

The two signs in Eqs. (2) and (6) refer to waves either propagating upward and downward (at an angle to the vertical) or evanescent in the direction of increasing or decreasing altitude. For positive $m^{2}$, the wave carries energy upward, if the upper sign is chosen in Eqs. (2) and (6) when $\omega_{d}^{2}>\Omega^{2}$ (i.e., on the acoustic branch of AGWs). Lower signs should be chosen when $\omega_{d}^{2}<N_{0}^{2}$ (i.e., on the gravity branch of AGWs) (Godin 2015). Here $\Omega$ and $N_{0}$ are the acoustic cutoff and smoothed buoyancy, or Brunt-Väisälä, frequencies:

$$
\Omega=\frac{\gamma g}{2 c}, \quad N_{0}=\sqrt{\gamma-1} \frac{g}{c}, \quad 0<N_{0}<\Omega .
$$

From the boundary condition $w=0$ at a stationary rigid surface $z=0$ and Eqs. (1) and (6), it follows that the ratio of pressures at the boundary of the downward propagating wave, $\tilde{p}_{i}$, and in the reflected, upward propagating wave, $\tilde{p}_{r}$, is 


$$
R=\frac{\tilde{p}_{r}}{\tilde{p}_{i}}=\exp \left[ \pm 2 i \arctan \left(\frac{g}{m}\left(\frac{k^{2}}{\omega_{d}^{2}}-\frac{\gamma}{2 c^{2}}\right)\right)\right]
$$

where the upper and lower signs correspond to propagating AGWs on the acoustic and gravity branches, respectively. Reflection coefficient of sound waves at a rigid surface is 1 (Brekhovskikh and Godin 1999). Equation (8) shows that, unlike sound, AGWs experience a phase shift at reflection from a rigid boundary.

In the atmosphere, waves are reflected and switch their direction of propagation with respect to vertical at the altitude where $m=0$. Within the WKB approximation, reflection at the turning point is accompanied by $\pi / 2$ phase loss (gain) on the acoustic and gravity branches, respectively (Godin 2016). Let $m\left(z_{t}\right)=0$ and $m>0$ at $0<z<z_{t}$. Then, away from the turning point $z_{t}$, on the acoustic branch we have (Godin 2016)

$$
\begin{aligned}
\tilde{p}(z)= & A \sqrt{\frac{\rho}{m}\left(\omega_{d}^{2}-\frac{g^{2} k^{2}}{\omega_{d}^{2}}\right)} \\
\times & \{\exp [i \varphi(z)+i \chi(z)] \\
& \left.-i \exp \left[2 i \varphi\left(z_{t}\right)+2 i \chi\left(z_{t}\right)-i \varphi(z)-i \chi(z)\right]\right\} .
\end{aligned}
$$

Here $A$ is a constant. On the gravity branch, $i$ should be replaced with $(-i)$ in Eq. (9).

\section{Resonance condition}

Atmospheric resonances occur when the wave launched by ground-level oscillations is reflected in the atmosphere, and the superposition of the direct and reflected waves satisfies the boundary condition on the rigid surface.

Let $m^{2}(z)>0$ at $0<z<z_{t}$, i.e., AGWs are propagating between the ground level and an elevated turning point. Calculating the wave-induced vertical displacement using Eqs. (6) and (9), we find that the boundary condition at $z=0$ is met when

$$
\begin{aligned}
\varphi\left(z_{t}\right)+\chi\left(z_{t}\right)=\pi\left(n-\frac{3}{4}\right) \\
-\arctan \left[\frac{g}{m(0)}\left(\frac{k^{2}}{\omega_{d}^{2}(0)}-\frac{\gamma}{2 c^{2}(0)}\right)\right] .
\end{aligned}
$$

Here $n=1,2, \ldots$ The resonance condition (10) applies to AGWs on both acoustic and gravity branches. Using Eq. (8) for the reflection coefficient from a rigid boundary, it is straightforward to verify that condition (10) has a simple physical meaning: a resonance occurs when waves with different numbers of reflections from the ground and the turning point interfere constructively. Indeed, Eq. (10) shows that the phase advance of a wave, which propagates upward, is reflected at $z=z_{t}$ and $z=0$, and returns to the original altitude, is $2 \pi(n-1)$.

Let the $x$ coordinate axis be chosen in the direction of the horizontal wave vector $\mathbf{k}$. Then $\mathbf{k}=(\omega / V, 0,0)$ and $\omega_{d}=\omega \beta, \quad \beta=1-u_{x} / V$, where $u_{x}$ is the $x$-component of the wind velocity. $V$ has the meaning of the trace velocity of the wave, i.e., the velocity with which phase of the wave propagates along the ground surface and any other horizontal plane. In terms of the trace velocity, Eq. (5) for the vertical component of the wave vector becomes

$$
m^{2}=\omega^{2}\left(\frac{\beta^{2}}{c^{2}}-\frac{1}{V^{2}}\right)-\frac{\gamma^{2} g^{2}}{4 c^{4}}+\frac{(\gamma-1) g^{2}}{\beta^{2} c^{2} V^{2}} .
$$

At any given altitude, for propagating $\left(m^{2}>0\right)$, as opposed to evanescent, AGWs $V$ is larger (smaller) than the effective sound speed $c+u_{x}$ on the acoustic (gravity) branch, respectively (Hines 1965; Gossard and Hooke 1975; Godin 2015).

Equation (11) shows that Eq. (10) is a characteristic equation that relates wave frequency and $V$. Solution $f_{n}(V)$ of the characteristic equation gives resonance frequency as a function of the trace velocity $V$ and resonance order $n$. Only the wind velocity component in the direction of the vector $\mathbf{k}$ enters the characteristic equation. For typical seismic and volcanic wave sources, $V$ is large compared to the wind velocity and sound speed in air. Then AGWs are on the acoustic branch, $m^{2}$ in Eq. (11) and the resonance frequency are insensitive to wind and the specific value of $V$. This resonance is known as the acoustic resonance. When $V$ is smaller than the effective sound speed $c+u_{x}$ at the ground level, Eq. (10) predicts that resonances occur on the gravity branch. We will refer to such solutions as buoyancy resonances. Solutions to Eq. (10) are illustrated in Figs. 1, 3, and 5 to be discussed later. The conditions of existence of the acoustic and buoyancy resonances and their properties in polar and mid-latitude atmosphere will be discussed in "Atmospheric resonances at high and mid-latitudes".

In the analysis presented above, we neglected the inertial effects resulting from Earth's rotation. This is justified when wave periods are small compared to the period $T_{e}=24 \mathrm{~h}$ of Earth's rotation. The inertial effects can be readily included in the WKB theory (Godin and Zabotin 2016 ) in the $f$-plane approximation (Gill 1982). However, the basic assumption of the stationary environment, i.e., of the temperature and wind profiles being independent of time, becomes questionable for long-period waves in the atmosphere. In this paper, we will disregard the inertial effects, which limits our analysis of buoyancy resonances to waves with periods less than about $6 \mathrm{~h}$. 


\section{Excitation of atmospheric waves}

Consider AGW excitation by vertical motion of the lower boundary of the atmosphere, which can represent ocean surface, ground, or an ice shelf. Here again the lower boundary of the atmosphere is treated as a rigid surface, so that its motion is not affected by perturbations in the atmosphere. This idealization is justified by the large difference in densities of air and the medium (water, ice, or solid ground) at $z<0$.

Let the vertical displacement of the surface from its time-averaged position $z=0$ be

$$
w=w_{0} \exp (i \xi x-i \omega t),
$$

where $w_{0}$ is the amplitude of the monochromatic plane wave propagating along the boundary and generating atmospheric waves. The frequency and horizontal wave vector of the resulting AGWs will be $\omega$ and $\mathbf{k}=(\xi, 0,0)$, respectively. Using Eqs. (6) and (9) and imposing the boundary condition of continuity of the normal displacement at the boundary, we find $w(z)=w_{0} W(\xi, z)$, where

$$
\begin{aligned}
W(\xi, z)= & \sqrt{\frac{\rho_{0} m_{0}\left(\omega_{d 0}^{2}-g^{2} k^{2} / \omega_{d 0}^{2}\right)}{\rho m\left(\omega_{d}^{2}-g^{2} k^{2} / \omega_{d}^{2}\right)}} \\
& {\left[\frac{g k^{2}}{\omega_{d 0}^{2}}-\frac{1}{2 h_{0}}+i m_{0}-i B\left(\frac{g k^{2}}{\omega_{d 0}^{2}}-\frac{1}{2 h_{0}}-i m_{0}\right)\right]^{-1} } \\
& \times\left\{e^{i \varphi(z)+i \chi(z)}\left(\frac{g k^{2}}{\omega_{d}^{2}}-\frac{1}{2 h}+i m\right)\right. \\
& \left.-i B e^{-i \varphi(z)-i \chi(z)}\left(\frac{g k^{2}}{\omega_{d}^{2}}-\frac{1}{2 h}-i m\right)\right\} .
\end{aligned}
$$

Here subscript 0 indicates the values of respective quantities at $z=0$, and

$$
B=\exp \left[2 i \varphi\left(z_{t}\right)+2 i \chi\left(z_{t}\right)\right] .
$$

Dependence of $B, \phi$, and $\chi$ on $\xi$ is implied and not shown explicitly. Note that Eq. (13) gives $w(0)=w_{0}$, as expected.

When $\omega$ and $V=\omega / \xi$ satisfy the characteristic Eq. (10), we have resonance excitation. Then the quantity in the square brackets in the right side of Eq. (13) is zero, and Eq. (13) predicts infinite values of AGW displacement and velocity amplitudes at $z>0$. Finite AGW amplitudes at resonance are obtained if one accounts for AGW dissipation or otherwise model finite quality factor of the resonance by assigning either $V$ or $\omega$ a finite imaginary part.

The formally infinite wave amplitude at resonance indicates a strong amplification of the atmospheric wave generation around the resonance frequency and should be contrasted with the case, where there are no turning points, and the amplitude of a radiated wave is always finite.

Consider now a more realistic scenario, where vibrations of the lower boundary occur in a finite (with respect to coordinate $x$ ) region. Let the vertical displacement of the surface $z=0$ be

$$
w=w_{0}(x) \exp (-i \omega t) .
$$

The function $w_{0}(x)$ is supposed to be integrable and may have a finite support (i.e., have non-zero values only in a finite range of $x$ values), as is the case, e.g., for AGW radiation by an ice shelf or a wave packet of infragravity waves in the ocean. Under these assumptions, the boundary

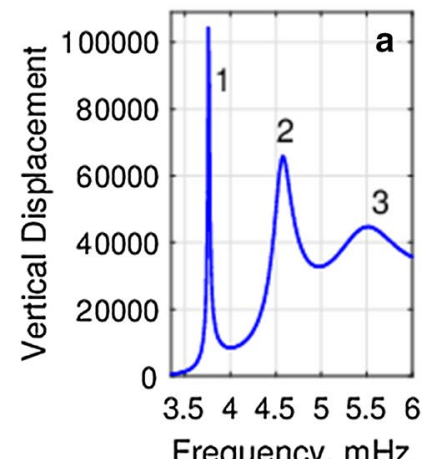

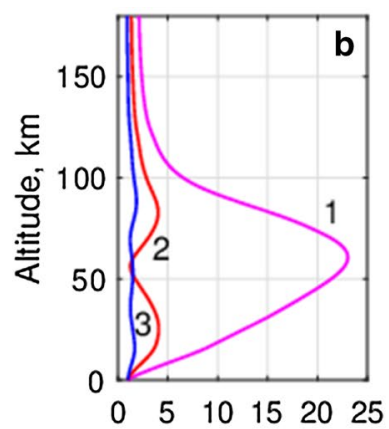

$\begin{array}{llllll}0 & 5 & 10 & 15 & 20 & 25\end{array}$

Reduced Vertical Displacement

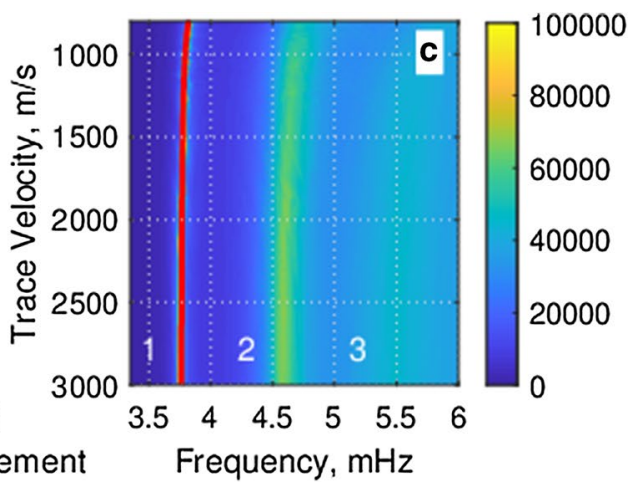

Frequency, $\mathrm{mHz}$

Fig. 1 Vertical displacements in a mid-latitude atmosphere due to vibrations of its lower boundary. a Displacement amplitude, $|w|$, at altitude $z=180 \mathrm{~km}$ in a wave with trace velocity $V=3000 \mathrm{~m} / \mathrm{s}$. Atmospheric resonances manifest as peaks of the displacement's frequency dependence. $\mathbf{b}$ Variation with altitude of the amplitude of the reduced vertical displacement Q, Eq. (23), at frequencies of the first three atmospheric resonances. c Variation of the resonance frequency and quality factor of acoustic resonances with the trace velocity of excitation. Color shows displacement amplitude at $z=180 \mathrm{~km}$. The red line is the WKB predictions for the frequency of the first acoustic resonance and is obtained from Eq. (10). Monochromatic ground-level displacements and summer conditions over Wallops Island (Virginia, USA) are assumed in all simulations. The displacement and reduced displacement amplitudes are normalized by their values at $z=0$ and are dimensionless. Resonance orders $n=1,2,3$ are indicated in all figures 
displacement amplitude can be represented as a Fourier integral:

$$
w_{0}(x)=\int_{-\infty}^{\infty} \exp (i \xi x) \Phi(\xi) \mathrm{d} \xi .
$$

Using the superposition principle, the solution for the vertical displacement in AGWs is easily found in terms of the spectrum $\Phi$ of the boundary displacement and the solution $W(\xi, z)$, Eq. (13), for plane-wave excitation:

$$
w(x, z)=\int_{-\infty}^{\infty} \exp (i \xi x) \Phi(\xi) W(\xi, z) \mathrm{d} \xi .
$$

The integrand in Eq. (17) becomes infinite at $\xi=\xi_{n} \equiv \omega /$ $V_{n}(\omega)$, where $V_{n}(\omega)$ are solutions of the characteristic Eq. (10). Resonance contributions to the AGW field can be calculated as contributions of poles at $\xi=\xi_{n}$ to the integral in Eq. (17), see, e.g., Brekhovskikh and Godin (1999). By calculating the residue in the respective pole, for the contribution of the $n$th resonance at $x>0$, we find

$$
\begin{aligned}
w_{n}(x, z)= & \sqrt{\frac{\rho_{0} m_{0}\left(\omega_{d 0}^{2}-g^{2} k^{2} / \omega_{d 0}^{2}\right)}{\rho m\left(\omega_{d}^{2}-g^{2} k^{2} / \omega_{d}^{2}\right)}}[D(\mathbf{k})]^{-1} \exp \left(i \xi_{n}|x|\right) \Phi\left(\xi_{n}\right) \\
& \times\left\{e^{i \varphi(z)+i \chi(z)}\left(\frac{g k^{2}}{\omega_{d}^{2}}-\frac{1}{2 h}+i m\right)\right. \\
& \left.-i B e^{-i \varphi(z)-i \chi(z)}\left(\frac{g k^{2}}{\omega_{d}^{2}}-\frac{1}{2 h}-i m\right)\right\} .
\end{aligned}
$$

Here $\mathbf{k}=(\xi x /|x|, 0,0)$ and

$$
\begin{aligned}
D & =\left(\frac { \partial } { \partial \xi } \left[\frac{g k^{2}}{\omega_{d 0}^{2}}-\frac{1}{2 h_{0}}+i m_{0}\right.\right. \\
& \left.\left.-i B\left(\frac{g k^{2}}{\omega_{d 0}^{2}}-\frac{1}{2 h_{0}}-i m_{0}\right)\right]\right)_{\omega} .
\end{aligned}
$$

In the WKB approximation, one should differentiate only the phase integral in $B$, Eq. (14), when calculating the derivative in Eq. (19) (Godin 2015). Then Eqs. (14) and (19) give an explicit equation for $D$ :

$$
\begin{aligned}
& D=-\left(m_{0}-i \frac{g k^{2}}{\omega_{d 0}^{2}}+\frac{i}{2 h_{0}}\right) \\
& \int_{0}^{z_{t}}\left\{\left[1-\frac{(\gamma-1) g^{2}}{c^{2} \omega_{d}^{2}}\right] k+\left[1-\frac{(\gamma-1) k^{2} g^{2}}{\omega_{d}^{4}}\right] \frac{\omega_{d} \mathbf{k} \cdot \mathbf{u}}{k c^{2}}\right\} \frac{\mathrm{d} z}{m} .
\end{aligned}
$$

We have considered a two-dimensional (2-D) excitation problem; the boundary displacement Eq. (15) and the resulting AGW field were assumed to be independent of the horizontal coordinate $y$. In 3-D problem, $w_{0}(x)$ in Eq. (15) is replaced with $w_{0}(x, y)$, and Fourier integrals in the counterparts of Eqs. (16) and (17) involve integration over both components of the horizontal wave vector $\mathbf{k}$. As in the 2-D problem, atmospheric wave field due to a finite source is expressed via the source spatial spectrum and the wave field $W(\mathbf{k}, z)$, Eq. (13), due to a plane-wave excitation. Analytical calculation of the resonance contribution to the 3-D wave field in the moving (windy) atmosphere in terms of known solutions $W(\mathbf{k}, z)$ of the 1-D (vertical) wave equation is discussed in (Brekhovskikh and Godin 1999). In the absence of winds, the 3-D resonant contribution in the far field of the source differs from the 2-D result Eq. (18) by replacement of $\exp \left(i \xi_{n} x\right)$ with the Hankel function $H_{0}^{(1)}\left(\xi_{n}\left(x^{2}+y^{2}\right)^{1 / 2}\right)$ and a factor describing source directionality in the horizontal plane, while winds contribute additional azimuthal anisotropy of the atmospheric wave field, see Brekhovskikh and Godin (1999).

\section{Atmospheric resonances at high and mid-latitudes Modeling of the atmosphere response to vibrations of its lower boundary}

Accuracy of the WKB theory is known to increase rapidly with increasing order of a resonance but the approximation has limited applicability to low-order resonances (Brekhovskikh and Godin 1999). Acoustic resonances of practical interest correspond to lowest values $n=1,2$, and possibly 3 in Eq. (10). Under these conditions, the height of the turning point is of the order of the vertical wavelength of the resonant AGW, and validity of the WKB approximation, which formally relies on the wave field varying with height much faster than the environment, is questionable. This fact was first pointed out by Jones and Georges (1976). Thus, it is necessary to verify the $\mathrm{WKB}$ results against an exact solution.

Consider linear AGWs with harmonic dependence on horizontal coordinates and time, where $w(x, y, z, t)=W(z) \exp (i \mathbf{k} \cdot \mathbf{r}-i \omega t)$, in a horizontally stratified atmosphere with time-independent parameters. The vertical displacement of fluid particles satisfies the 1-D wave equation (Godin 2015)

$$
\begin{aligned}
& \frac{\mathrm{d}}{\mathrm{d} z}\left[\frac{\rho \omega_{d}^{2} d W / \mathrm{d} z}{\omega_{d}^{2} c^{-2}-k^{2}}\right] \\
& \quad+\left[\omega_{d}^{2}-\frac{g^{2} k^{2}}{\omega_{d}^{2}-k^{2} c^{2}}-\frac{g}{\rho} \frac{\mathrm{d}}{\mathrm{d} z}\left(\frac{\rho k^{2}}{\omega_{d}^{2} c^{-2}-k^{2}}\right)\right] \rho W=0
\end{aligned}
$$


as well as a boundary condition at the ground level $(z=0)$ and a radiation condition at high altitudes (at $z \rightarrow+\infty)$. The radiation condition ensures that the wave field either vanishes at or transports energy towards $z \rightarrow+\infty$.

Because of the exponential decrease of the air density $\rho$ with altitude, coefficients in Eq. (21) may vary by several orders of magnitude between the ground level and upper atmosphere, and the solution $W$ may increase exponentially with $z$. Numerical solution of the wave equation is simplified if Eq. (21) is cast in an equivalent form

$$
\begin{aligned}
\frac{\mathrm{d}}{\mathrm{d} z} & \left\{\frac{\omega_{d}^{2}}{\omega_{d}^{2} c^{-2}-k^{2}}\left[\frac{\mathrm{d} Q}{\mathrm{~d} z}+\frac{Q}{2 h}\left(1+\frac{\mathrm{d} h}{\mathrm{~d} z}\right)\right]\right\} \\
& -\frac{1}{2 h}\left(1+\frac{\mathrm{d} h}{\mathrm{~d} z}\right) \frac{\omega_{d}^{2}}{\omega_{d}^{2} c^{-2}-k^{2}}\left[\frac{\mathrm{d} Q}{\mathrm{~d} z}+\frac{Q}{2 h}\left(1+\frac{\mathrm{d} h}{\mathrm{~d} z}\right)\right] \\
& +\left\{\omega_{d}^{2}+\frac{g k^{2}}{\omega_{d}^{2} c^{-2}-k^{2}}\left[\frac{1}{h}\left(1+\frac{\mathrm{d} h}{\mathrm{~d} z}\right)-\frac{g}{c^{2}}+\frac{\mathrm{d}}{\mathrm{d} z} \ln \left(\omega_{d}^{2} c^{-2}-k^{2}\right)\right]\right\} Q=0
\end{aligned}
$$

in terms of the reduced vertical displacement

$$
Q(z)=\sqrt{\rho(z) / \rho(0)} W(z) .
$$

In Eq. (23), the displacement is normalized to compensate for the density-related exponential increase of its amplitude with altitude. Unlike Eq. (21), there are no exponentially small and exponentially large coefficients in Eq. (22), which makes it more suitable for solving numerically. Since $Q=W$ at $z=0$, the boundary condition for the reduced displacement at a stationary or vibrating rigid ground surface is the same as for $W$.

Assuming that AGW reflection in the upper atmosphere becomes negligible at altitudes above certain altitude $H$, Eq. (22) needs to be solved numerically only in a finite interval $0<z<H$. Details of wind velocity and temperature profiles at $z>H$ have no effect on the AGW field at $z<H$ and on occurrence of the resonances. We model the non-reflecting part of the upper atmosphere as an isothermal, uniformly moving half-space $z>H$, with the wind velocity and air temperature at $z=H$ being extrapolated to $z>H$.

The scale height $h$, sound speed $c$, intrinsic frequency $\omega_{d}$, and vertical component of the wave vector $m$ Eq. (5) are all constant in an isothermal, uniformly moving halfspace. Then the wave Eq. (22) simplifies and has linearly independent solutions $Q=\exp ( \pm i m z)$. For evanescent waves, where $m=i|m|$, and propagating AGWs on the acoustic branch, only solutions $Q=$ const. $\times \exp (i m z)$ meet the radiation condition. With $Q$ known at $z>H$, the conditions of continuity of Lagrangian pressure perturbation and vertical displacement at $z=H$ (Godin 1997) can be written as the one-sided boundary condition

$$
\frac{1}{Q} \frac{\mathrm{d} Q}{\mathrm{~d} z}=i m-\frac{1}{2 h} \frac{\mathrm{d} h}{\mathrm{~d} z}, \quad z=H-0
$$

for the wave field at $z<H$. Note that $h^{-1} \mathrm{~d} h / \mathrm{d} z$ in the right side of Eq. (24) equals the value of the logarithmic derivative, $T^{-1} \mathrm{~d} T / \mathrm{d} z$, of the absolute temperature, $T$, just below the interface $z=H$. Equation (23) and the relation (Godin 2015)

$$
\tilde{p}=\rho\left(\frac{k^{2}}{\omega_{d}^{2}}-\frac{1}{c^{2}}\right)^{-1}\left(\frac{\partial w}{\partial z}-\frac{g k^{2}}{\omega_{d}^{2}} w\right)
$$

between the vertical displacement and pressure perturbation were used in derivation of the boundary condition (24).

To model AGW field, wave Eq. (22) was solved numerically at $0 \leq z<H$ with the initial conditions

$$
Q_{1}=1, \quad \frac{\mathrm{d} Q_{1}}{\mathrm{~d} z}=i m-\frac{1}{2 h} \frac{\mathrm{d} h}{\mathrm{~d} z}, \quad z=H-0,
$$

for a set of wave frequencies $\omega$ and horizontal wave vectors $\mathbf{k}$. The initial conditions (26) ensure that the radiation condition is satisfied. In terms of the numerical solution $Q_{1}(z)$, the vertical displacement in the wave generated by the lower boundary vibrations with the vertical displacement amplitude $w_{0}$ is given by

$$
w=w_{0} \sqrt{\frac{\rho(0)}{\rho(z)}} \frac{Q_{1}(z)}{Q_{1}(0)} \exp (i \mathbf{k} \cdot \mathbf{r}-i \omega t)
$$

The vertical component of the power flux density in AGWs is (Godin 1997)

$$
I_{z}=p \partial w / \partial t
$$

Using Eqs. (25) and (28), for the time-averaged vertical component of the power flux density in a monochromatic wave we find

$$
I_{z}=\frac{\omega}{2} \operatorname{Im}\left(p^{*} w\right)=-\frac{\omega \rho}{2}\left(\frac{k^{2}}{\omega_{d}^{2}}-\frac{1}{c^{2}}\right)^{-1} \operatorname{Im}\left(w^{*} \frac{\mathrm{d} w}{\mathrm{~d} z}\right) .
$$

Here and below asterisk denotes complex conjugation. With the vertical displacement given by Eq. (27), Eq. (29) becomes

$$
I_{z}=-\frac{\omega \rho(0)}{2}\left|\frac{w_{0}}{Q_{1}(0)}\right|^{2}\left(\frac{k^{2}}{\omega_{d}^{2}}-\frac{1}{c^{2}}\right)^{-1} \operatorname{Im}\left(Q_{1}^{*} \frac{\mathrm{d} Q_{1}}{\mathrm{~d} z}\right)
$$

In an atmosphere without dissipation, $I_{z}$ is constant, and the right side of Eq. (30) should be independent of $z$. 
This property was used to verify the validity and accuracy of the numerical solutions $Q_{1}(z)$.

In the first WKB approximation, the vertical power flux density Eq. (29) in the wave launched upwards by oscillations of the lower boundary is

$$
I_{\text {in }}=\left.\frac{\omega \rho m}{2}\left(\frac{1}{c^{2}}-\frac{k^{2}}{\omega_{d}^{2}}\right)^{-1}\right|_{z=0}\left|w_{0}^{2}\right| .
$$

We assume here that $m^{2}>0$ at $z=0$. The total vertical power flux density is generally less than $I_{\text {in }}$ because of the downward power flux in reflected waves. $I_{\mathrm{z}}=0$ at a perfect resonance when all radiated energy is reflected back to the ground level. When a fraction of energy escapes and is radiated towards $z \rightarrow+\infty$, the ratio

$$
r \equiv \frac{I_{z}}{I_{\text {in }}}=\left.\frac{1}{m(0)} \operatorname{Im}\left(\frac{1}{Q_{1}} \frac{\mathrm{d} Q_{1}}{\mathrm{~d} z}\right)\right|_{z=0}
$$

quantifies radiation losses. The ratio takes values $0 \leq r \leq 1$. In the WKB approximation $r=1$ in the absence of a turning point, and $r=0$ when $m^{2}<0$ at all altitudes above the turning point.

Consider an atmosphere where, in a certain range of $\omega$ and $k$, there are propagating AGWs at $0<z<z_{t 1}$ and $z>z_{t 2}$, while the waves are inhomogeneous (evanescent) at $z_{t 1}<z<z_{t 2}$. If an AGW is launched from the ground level, it will be partially reflected around the lower turning point $z_{t 1}$ and will also generate a propagating wave above the upper turning point $z_{t 2}$. The latter wave will propagate towards increasing $z$, i.e., upwards. Between the turning point altitudes, AGW field is a superposition of two evanescent waves with the opposite signs of their (imaginary) vertical component of the wave vector. Similar to the quantum mechanical counterpart of this problem, transmission of wave energy through the "potential barrier" $z_{t 1}<z<z_{t 2}$ will be referred to as tunneling. In application to acoustic resonances, the tunneling proves to be very important for two reasons. First, the "potential barrier" is typically not thick on the scale of AGW vertical wavelength (see "Acoustic resonances"), which makes it penetrable. Second, as discussed in "Introduction", most of the reported observations of atmospheric resonances are related to their manifestations at ionospheric altitudes at $z>z_{t 2}$. The strength of tunneling is controlled by the absolute value of the phase integral, $\left|\varphi\left(z_{t 2}\right)-\varphi\left(z_{t 1}\right)\right|$, between the turning points, see, e.g., Godin and Naugolnykh (2005) for an analysis of tunneling of infrasound in the atmosphere. In this paper, AGW tunneling will be quantified using numerical solutions of Eq. (22).

\section{Acoustic resonances}

We illustrate properties of acoustic resonances of a mid-latitude atmosphere and their tunneling to upper atmosphere by modeling AGWs under conditions characteristic of Wallops Island $\left(37.94{ }^{\circ} \mathrm{N} 75.47{ }^{\circ} \mathrm{W}\right)$, Virginia, USA, where NASA's Wallops Island Flight Facility is located. Observations of AGWs at this location with a Dynasonde HF radar system have been previously described in Godin et al. (2015) and Zabotin et al. (2016). Figure 1 presents results of numerical modeling of atmospheric oscillations generated by a periodic displacement of the boundary $z=0$. Air temperature profile in summer atmosphere (specifically, for 14 July 2014) was generated by the atmospheric model NRLMSISE-00.2 (Picone et al. 2002) (Fig. 2). Profiles of the wind velocity components were generated using NRL's Horizontal Wind Model HWM14 (Drob et al. 2015). In the AGW simulations, the trace velocity of the boundary vibrations was given values larger than the sum of the wind velocity and sound speed in air at the ground level, so that near the boundary, propagating (as opposed to evanescent) AGWs could be generated only on the acoustic branch.

As a function of frequency, response of the upper atmosphere (Fig. 1) and all the way down to the troposphere showed strong increases at several frequencies in the $\mathrm{mHz}$ range. The first, lowest frequency resonance, at Wallops Island occurs at frequencies from about $3.74 \mathrm{mHz}$ to $3.88 \mathrm{mHz}$ depending on the trace velocity. The second and third acoustic resonances have frequencies from about 4.60 to $4.75 \mathrm{mHz}$ and $5.4-6.0 \mathrm{mHz}$, respectively. The resonance frequencies decrease with increasing trace velocity. The same trend is predicted by the WKB approximation (Eq. (10)), see Fig. 1c. The vertical dependence of the reduced displacement in the $n$th resonance, $n=1,2,3$, has $n$ maxima (Fig. 1b) in agreement with the WKB Eqs. (10) and (13). The asymptotic result for the frequency of the first resonance, which is shown by the red curve in Fig. 1c, reproduces the numerical result very accurately. For the second and third resonance, AGWs prove to be evanescent near the boundary $z=0$ under the specific atmospheric conditions considered. Then the asymptotic resonance condition Eq. (10), which assumes that $m^{2}(0)>0$, cannot be used to calculate the resonant frequencies. Numerical solution of the wave equation (Fig. 1) shows, however, that appearance of the additional, low-altitude turning point does not prevent occurrence of the acoustic resonances.

The quality factor of the resonances, which is inversely proportional to their frequency extent (at fixed $V$ ) and directly proportional to the resonant amplification of 

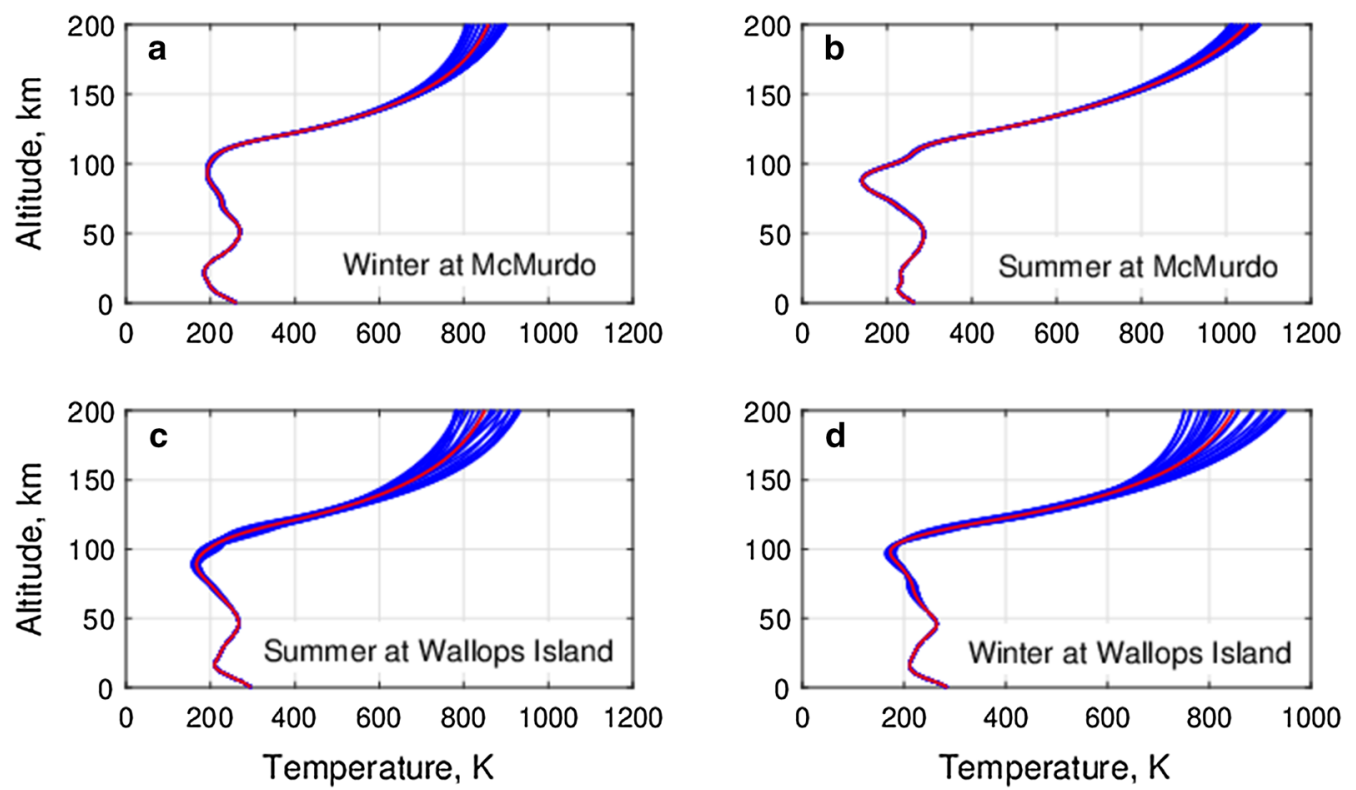

Fig. 2 Summer and winter atmospheric conditions at McMurdo (Antarctica) and Wallops Island (Virginia, USA). Hourly absolute temperature profiles generated using the atmospheric model NRLMSISE-00.2 (Picone et al., 2002) are shown by blue lines for a 14 July 2014 (winter) at McMurdo; b 15 January 2014 (summer) at McMurdo; c 14 July 2014 (summer) at Wallops Island; and d 15 January 2014 (winter) at Wallops Island. Time averages of the hourly temperature profiles are shown in red

wave amplitude, rapidly decreases with increasing order of acoustic resonances (Fig. 1a, c). When AGW absorption at altitudes below the upper turning point $z_{t 2}$ is negligible, the quality factor is limited only by radiation losses due to AGW tunneling. As the order of the resonance increases, the turning points approach the top of the "potential barrier," i.e., the minimum of $m^{2}(z)$, the barrier becomes thinner and lower, and reflection from the barrier becomes weaker. This can be also seen in the vertical dependence of the reduced displacement (Fig. 1b). In the case of total reflection from the lower turning point $z_{t \mathbf{1}}$, amplitudes of the waves propagating up- and downward are equal at $z<z_{t 1}$, and the displacement equals zero at interference minima. According to Eqs. (10) and (13) the first resonance does not have an interference minimum at $z>0$, in agreement with Fig. 1b. Interference minima of the vertical dependence of the reduced displacement in the second and third resonances in Fig. $1 \mathrm{~b}$ do not extend to zero because of only partial AGW reflection from the "potential barrier." The ratio of the values of the reduced displacement amplitudes at the interference minima and maxima can serve as a measure of the fraction of the AGW power flux that is radiated into the upper atmosphere above the upper turning point in respective resonant oscillations.

Comparison of the results of simulations in atmospheres with and without wind shows that properties of the acoustic resonances, including their frequencies, are insensitive to wind, especially at large trace velocities. This is a consequence of the trace velocities being large for ground level vibrations that excite acoustic resonances. As discussed above in connection with Eq. (11), wind velocity enters the wave equation and boundary conditions via the intrinsic frequency $\omega_{d}=\omega \beta, \quad \beta=1-u_{x} / V$, where $u_{x}=\mathbf{k} \cdot \mathbf{u} / k$ is the projection of wind velocity on the direction of the horizontal wave vector. Obviously, the wind effect is negligible when $\left|u_{x}\right| \ll V$.

Figure 1 refers to summer conditions. Simulations' results obtained for winter conditions at the Wallops Island (Fig. 2d) prove to be similar and are not shown.

Consider now excitation of a polar atmosphere oscillations by vibrations of its lower boundary, which may be caused, e.g., by seismic waves propagating over solid ground or vibrations by large ice shelves. As an example of a high-latitude environment, we choose McMurdo, Antarctica $\left(77.84{ }^{\circ} \mathrm{S}, 166.67{ }^{\circ} \mathrm{E}\right)$, because of the previously reported observations (Chen et al. 2016) of persistent, large-amplitude atmospheric waves at this location. To generate air temperature and wind velocity profiles at McMurdo we again used atmospheric models NRLMSISE-00.2 (Picone et al. 2002) and HWM14 (Drob et al. 2015). Results of numerical modeling of atmospheric oscillations at McMurdo, which are generated by periodic displacement of the boundary $z=0$ with supersonic trace velocities, are illustrated in Fig. 3. Figure 3 refers to 
the atmospheric conditions at noon on 15 January 2014 during the Southern hemisphere summer. Diurnal variations of the atmosphere (Fig. 2b) proved to have a negligible effect on the forced oscillations illustrated in Fig. 3 and are not shown there.

The acoustic resonances of summer atmosphere at McMurdo (Fig. 3) are similar to resonances at Wallops Island (Fig. 1). Depending on the trace velocity of the excitation, the first three resonances occur at frequencies approximately $3.64-3.79,4.55-4.80$, and 5.6-6.0 $\mathrm{mHz}$. Resonance frequencies of the first two resonances are within $0.1 \mathrm{mHz}$ of the values found for mid-latitude atmosphere at Wallops Island. The third resonance has a small quality factor and is poorly defined at McMurdo although not quite as poorly as at Wallops Island. Resonant frequencies increase with decreasing trace velocity (Fig. 3c). The "potential barrier," which is responsible for strong AGW reflection back to the ground, is located around the mesopause (at $z_{t p} \approx 90 \mathrm{~km}$ altitude in Fig. 2b). For each of the acoustic resonances, turning points occur above and below the mesopause; $z_{t 1}<z_{t p}<z_{t 2}$. The WKB resonance condition Eq. (10) rather accurately predicts the frequencies of the first two resonances, for which $m^{2}(0)>0$. The agreement with exact numerical solutions of the wave equation would not be possible without taking into account the Berry phase and the AGW phase shift at reflection from a rigid boundary in the asymptotic resonance condition Eq. (10).

While the first resonance is predicted to provide more than an order-of-magnitude amplification in a narrow frequency band and the second resonance is also well defined both in terms of the significant amplification and frequency selectivity (Fig. 3a, c), the third resonance would be much harder to identify in observations, especially when the power spectrum of the excitation is not flat.

The differences between the vertical structure of resonant wave fields at mid- and high latitudes is more pronounced than the difference in resonance frequencies (cf. Figs. $1 \mathrm{~b}$ and $3 \mathrm{~b}$ ). When excited at their resonant frequencies with the same amplitude at the ground level, the first and second resonances at McMurdo have higher peak amplitudes of the reduced vertical displacements and stronger manifestations at altitudes above $150 \mathrm{~km}$ than the corresponding atmospheric resonances at Wallops Island.

It should be emphasized that, because of tunneling through the "potential barrier," amplitudes of the reduced displacement $Q(z)$, Eq. (23), have significant values and decrease only slightly above the upper turning point of the respective resonance (see Figs. $1 \mathrm{~b}$ and $3 \mathrm{~b}$ ) The vertical displacement $W(z)$ increases exponentially above the upper turning point and reaches very high values (Figs. 1a and 3a).

Unlike the summer conditions discussed above, no acoustic resonances of atmospheric oscillations are found under winter conditions at McMurdo (Fig. 2a). The characteristic Eq. (10) has no solutions for any supersonic trace velocities $V$ that result in the occurrence of a turning point. This can be traced back to a specific seasonal change in the thermal structure of the polar atmosphere, namely the absolute minimum of temperature occurs at the mesopause in summer and at the tropopause in winter.

As has been already pointed out, winds have a small effect on acoustic resonances at large supersonic trace velocities and, therefore, can be neglected in a qualitative discussion. Then existence and altitudes of turning points are determined by the sound speed profile. It follows from the AGW dispersion relation Eq. (11) that,
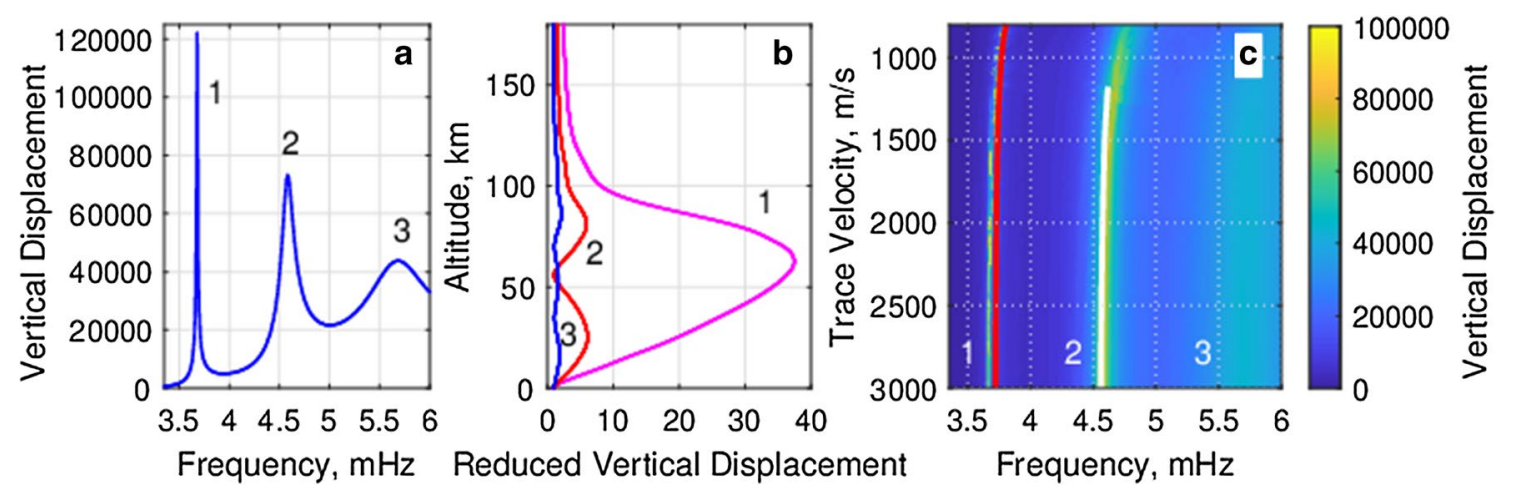

Fig. 3 Vertical displacements in a high-latitude atmosphere due to vibrations of its lower boundary. Same as in Fig. 1 but summer conditions over McMurdo (Antarctica) are assumed in all simulations. The WKB predictions for the frequencies of the first and second acoustic resonance are shown in (c) by the red and white lines, respectively 
at the low frequencies representative of the acoustic resonances, $m^{2}$ decreases with decreasing sound speed and, hence, decreasing temperature. (This is in contrast to the high-frequency behavior, where $m^{2}$ increases with decreasing sound speed as is does for sound). In the acoustic resonance, an upward propagating AGW is reflected back to the ground from a turning point in the mesosphere due to negative temperature gradient. For an AGW to propagate from the ground level to the mesosphere and back to the ground, temperature at the mesospheric turning point needs to be lower than in the tropopause. This is the case at Wallops Island (Fig. 2c, d) and at McMurdo in summer (Fig. 2b). During Antarctic winter, when temperature at the tropopause is lower than at the mesopause, the AGWs, which would have a turning point in the mesosphere, are reflected in the troposphere on their way up from the ground level. With the turning point occurring at relatively low altitude, the AGW phase advance on its way from the ground level to the turning point, $\phi\left(z_{t}\right)+\chi\left(z_{t}\right)$, proves too small to satisfy the resonance condition Eq. (10) and, hence, for the acoustic resonance to form.

The discussion in "Asymptotic theory of atmospheric resonances" and the calculations illustrated in Figs. 1 and 3 do not take into account wave dissipation in the atmosphere. Using the theories (Golitsyn 1965; Godin 2014) of AGW dissipation due to air viscosity and thermal conductivity, we have evaluated the wave dissipation for the acoustic resonances shown in Figs. 1 and 3. Dissipation causes losses of less than $0.1 \mathrm{~dB}$ on the round-trip propagation path between the ground level and the lower turning point. The dissipated energy is small compared to the wave energy radiated into the upper atmosphere as a result of the AGW tunneling. Thus, the effect of wave dissipation on acoustic resonances was found to be negligible under the mid-latitude and polar atmospheric conditions considered in this paper.

\section{Buoyancy resonances}

When the trace velocity $V$ is subsonic (more precisely, less than the sum of the sound speed and the projection of the wind velocity on the direction of the horizontal wave vector $\mathbf{k}$ ), propagating AGWs belong to the buoyancy (also referred to as gravity) branch and have intrinsic frequencies below the smoothed buoyancy, or Brunt-Väisälä, frequency $N_{0}$, see Eq. (7) (Gossard and Hooke 1975; Godin 2015). Using the asymptotic theory [Eq. (9)], Fig. 4 illustrates response of the summer midlatitude atmosphere (Fig. 2c) to the excitation with a subsonic trace velocity (Fig. 4a, b) and of the summer polar atmosphere (Fig. 2b) to harmonic excitation of its lower boundary with a supersonic trace velocity (Fig. 4c).
Amplitude of the reduced vertical displacement, as given by Eqs. (13) and (23), is shown in Fig. 4 between the ground level and AGW turning point. Interference pattern between an upward propagating AGW and the downward propagating AGW reflected from the turning point is clearly visible and represents a standing wave. Atmospheric resonances manifest in the figure as a large increase of the displacement amplitude in a narrow frequency band. Acoustic and buoyancy resonances occur in distinct frequency bands but are qualitatively similar in terms of the altitude and frequency dependencies of the wave field. Figure 4 clearly shows that the resonances emerge when a null of the interference pattern approaches the ground level. The number of the nulls equals the order $n$ of the resonance in Eq. (10). Unlike acoustic resonances (Figs. 1, 3, and 4c), buoyancy resonances are not restricted to the lowest orders $1 \leq n \leq 3$ and can have a higher order $n$ (Fig. $4 \mathrm{a}, \mathrm{b}$ ). Note that, in contrast to acoustic resonances, some buoyancy resonances in Fig. 4 have their lowest turning point above the mesopause and can propagate to thermospheric altitudes without tunneling.

Numerical simulations show that the frequencies and the vertical structure of buoyancy resonances are much more sensitive than of acoustic resonances to diurnal and other moderate variations of the temperature profile. In terms of the AGW dispersion equation, it can be traced back to the terms with $k^{2}$ in the right side of Eq. (5) being no longer small compared to $\omega_{d}^{2} / c^{2}$ and $\gamma^{2} g^{2} / c^{4}$. Buoyancy resonances are also much more sensitive to the wind velocity profile. Ultimately, the increased sensitivity is due to the fact that the trace velocity $V$ is smaller for the buoyancy resonances than for acoustic ones and can be comparable to the wind speed. Relative changes in the wind velocity are typically much larger and tend to occur on shorter timescales than changes in the absolute temperature, which also contributes to temporal variability of the buoyancy resonances. Moreover, sensitivity to the wind velocity profile leads to anisotropy of the atmospheric response in the horizontal plane. For a given wind velocity profile and trace velocity magnitude, resonance frequencies depend on the direction of the horizontal wave vector, see Eq. (5), where wave propagation direction enters via the intrinsic frequency $\omega_{d}=\omega \beta, \quad \beta=1-k^{-1} \mathbf{k} \cdot \mathbf{u} / V$. Comparison of Fig. 4a, $\mathrm{b}$ shows that the resonance frequencies and even the number of the excited buoyancy resonances are different for different directions of propagation of the excitation along the lower boundary of the atmosphere. The altitudes of the turning points also change significantly (Fig. 4a, b). The trace velocity, $V=200 \mathrm{~m} / \mathrm{s}$, in Fig. 4a, b is representative of the tsunami speed in the open ocean, and the figures illustrate that modeling of ionospheric 

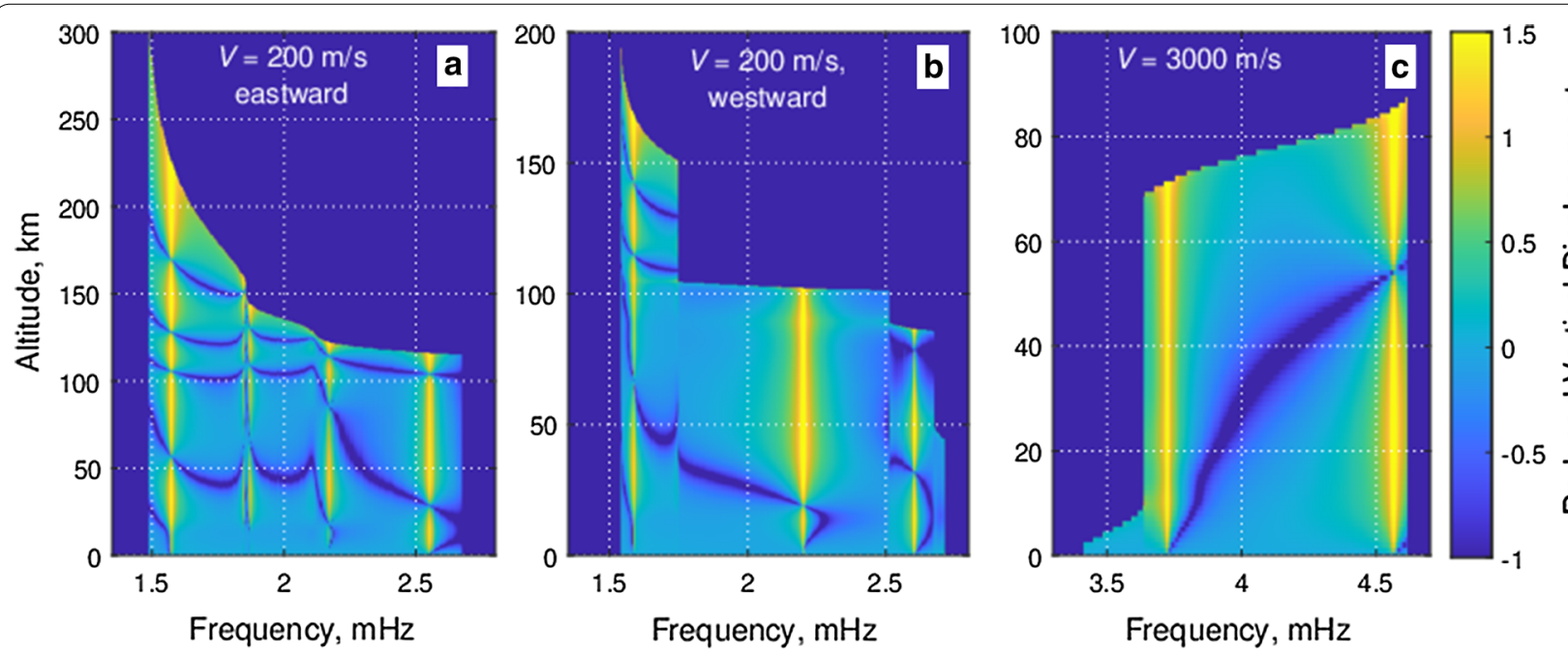

Fig. 4 Response of the atmosphere to monochromatic vibrations of its lower boundary. The amplitude of the reduced vertical displacement is shown on logarithmic scale, $\log _{10}|Q(z) / Q(0)|$, as a function of altitude and the frequency of oscillations. Displacement amplitude is normalized by its value at the ground level. Atmospheric waves are excited by vibrations that travel a eastward with subsonic trace velocity $V=200 \mathrm{~m} / \mathrm{s} ; \mathbf{b}$ westward with subsonic trace velocity $V=200 \mathrm{~m} / \mathrm{s}$; and c southward at supersonic trace velocity V=3000 m/s. Conditions at Wallops Island, Virginia, on 14 July 2014 are assumed in $\mathbf{a}$ and $\mathbf{b}$; conditions at McMurdo, Antarctica, on 15 January 2014 are assumed in c

effects of tsunamis without account for wind (Rolland et al. 2011) can reproduce the observed effects only qualitatively.

Dependence of the frequencies of buoyancy resonances on trace velocity is illustrated in Fig. 5 for winter and summer temperate atmosphere. Two times of day and two directions of wave propagation are shown in each of the two seasons. The background atmospheric conditions at Wallops Island, Virginia, are simulated using the NRLMSISE-00.2 (Picone et al. 2002) and HWM14 (Drob et al. 2015) models. Under the atmospheric conditions considered, buoyancy resonances can have turning points in (and, hence, extend from the ground level to) a wide range of mesospheric and thermospheric altitudes (Fig. 5). AGW absorption (Golitsyn 1965; Godin 2014) on the propagation path from the ground level the turning point typically increases and the quality factor of the resonance decreases with increasing turning point altitude. Resonances having a low quality factor are hardly observable. Figure 5 shows only well-defined resonances with the absorption not exceeding $1 \mathrm{~dB}$ on the path from the atmosphere's lower boundary to the turning point, where the wave is reflected back to the ground level. The resonance frequencies are predicted using the asymptotic resonance condition Eq. (10). The resonances having two or more turning points, which are not reliably described using Eq. (10), are not shown. No resonances exist at the highest subsonic trace velocities above about $250 \mathrm{~m} / \mathrm{s}$ (Fig. 5).
As for the acoustic resonances, a verification of the asymptotic theory's predictions of the buoyancy resonances can be obtained by a direct numerical solution of the AGW wave Eq. (21) with boundary conditions (26). In contrast to acoustic resonances, the oscillations that excite buoyancy resonances travel with subsonic, rather than supersonic, speeds along the lower boundary of the atmosphere. At such trace velocities, a resonant response of the atmosphere proves to occur, when AGWs are evanescent at high altitudes. Physically, it implies that, in contrast to acoustic resonances, there is no tunneling. Mathematically, for the evanescent waves, $i m=-|m|$ in the second boundary condition in Eq. (26).

Results of the direct numerical simulations of the response of a temperate atmosphere to the excitations with subsonic trace velocities are illustrated in Fig. 6. In the figure, atmospheric resonances manifest themselves as sharp increases of the oscillation amplitude in the upper atmosphere at a discrete set of frequencies for a given trace velocity $V$ (cf. Figs. 1c and 3c for the acoustic resonances). Positions of the resonance frequencies at different trace velocities form lines, which correspond to solid lines in Fig. 5a. AGW dissipation is not accounted for in the wave Eq. (21). Therefore, resonance frequencies appear in Fig. 6 in a wider frequency band and up to higher resonance order than in Fig. 5a, which shows only well-defined resonances obtained under the same atmospheric conditions but with the dissipation taken into account. In addition to lines with a strong amplitude 

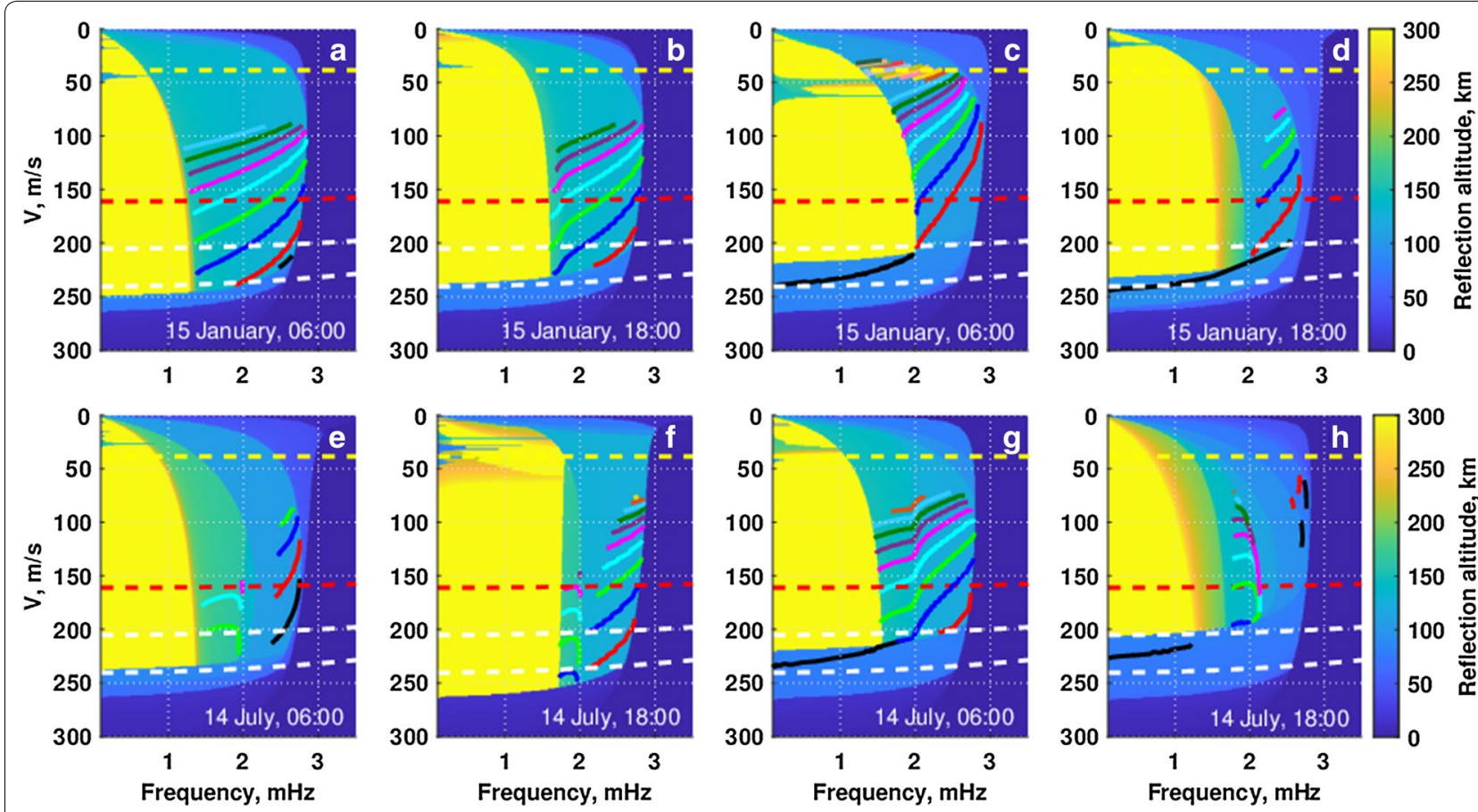

Fig. 5 Predicted buoyancy resonances of a mid-latitude atmosphere and their variability. Frequencies of buoyancy resonances of orders $n$ from 1 to 15 are shown by solid lines, with black lines corresponding to $n=1$, in a wide range of trace velocities. Color scale represents the altitude of AGW turning point. The overlaid dashed lines show dispersion curves of infragravity waves in the ocean with depth of $150 \mathrm{~m}$ (yellow), $2649 \mathrm{~m}$ (red), $4300 \mathrm{~m}$ (top white) and $5900 \mathrm{~m}$ (bottom white). The background atmospheric temperature and wind velocity profiles used in the simulations are obtained for Wallops Island, Virginia, with the NRLMSISE-00.2 (Picone et al. 2002) and HWM14 (Drob et al. 2015 ) models for two specific dates corresponding to winter and summer seasons: 15 January 2014 (a-d) and 14 July 2014 (e-h). For each date two times of the day, GMT 06:00 (a, c, e, $\mathbf{g}$ ) and GMT 18:00 (b, d, f, h), and two directions of the wave propagation, eastward (a, b, e, f) and westward (c, d, $\mathbf{g}, \mathbf{h})$ are shown

amplification and a high frequency selectivity, there are wider bands with smaller peak amplitudes, which extend to lower frequencies in Fig. 6. These bands represent resonances with a low quality factor and are apparently caused by the partial reflections in the atmosphere that are not captured in the asymptotic theory. With these caveats, comparison of Figs. 5a and 6 shows a qualitative agreement between the asymptotic theory and numerical results for the lowest order resonances and a very good quantitative agreement for resonances of the order $n>3$.

The resonance order $n$, the number of buoyancy resonances, and resonant frequency tend to increase with decreasing trace velocity (Figs. 5 and 6). With a possible exception for the $n=1$ resonance, well-defined buoyancy resonances are found in Fig. 5 at frequencies from $1 \mathrm{mHz}$ to below $3 \mathrm{mHz}$. The lower bound of the frequency band depends on the season and the time of day, see Fig. 5, where Fig. $5 \mathrm{a}-\mathrm{d}$ and $\mathrm{e}-\mathrm{h}$ refers to the January and July conditions, respectively.

The lower bound depends also on the time of day. Comparison of Fig. $5 \mathrm{a}$ with $5 \mathrm{~b}, 5 \mathrm{c}$ with $5 \mathrm{~d}, 5 \mathrm{e}$ with $5 \mathrm{f}$, and $5 \mathrm{~g}$ with $5 \mathrm{~h}$ illustrates the extent of diurnal variations of the buoyancy resonances. The first figure in each pair is

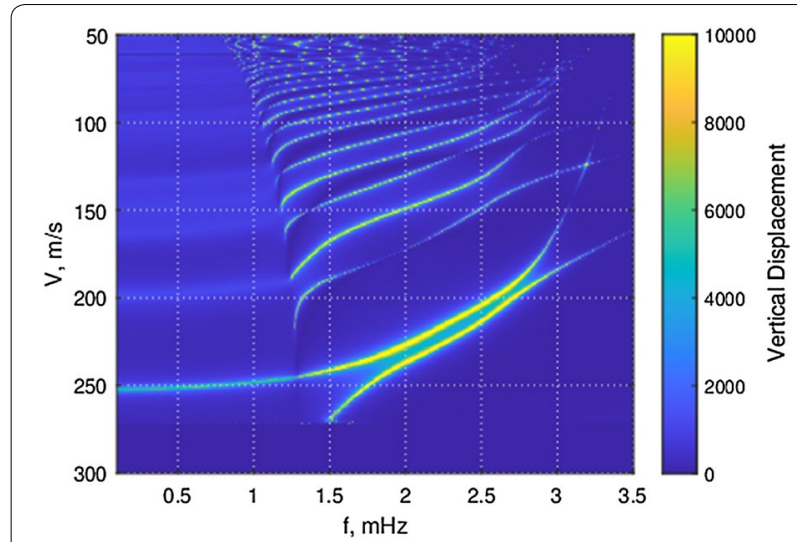

Fig. 6 Full-wave simulations of the oscillations in the thermosphere due to ground-level vibrations. Oscillations are generated by monochromatic vertical displacements of frequency $f$ traveling with the subsonic velocity $V$ along the lower boundary of the atmosphere. Color shows the amplitude of the vertical displacement at $z=180 \mathrm{~km}$. The displacement amplitudes are normalized by their values at $z=0$ and are dimensionless. The simulation assumes the same winter atmospheric conditions over Wallops Island (Virginia, USA) and propagation direction of the excitation as in Fig. 5a 
obtained for conditions at GMT 06:00, and the second one-at GMT 12:00 on the same day. Diurnal variations of the background atmospheric conditions prove to have a strong effect on the buoyancy resonances, including the number of the well-defined resonances (Fig. 5e, f), the range of their trace velocities (Fig. $5 \mathrm{c}, \mathrm{d}$ ), resonant frequencies of particular resonances, and the frequency range, where resonances occur (Fig. $5 \mathrm{~g}$, h). Our simulations suggest that the strongest diurnal effects tend to occur for higher order resonances and at trace velocities below about $100 \mathrm{~m} / \mathrm{s}$, with diurnal variability being stronger in summer (Fig. 5e-h) than in winter (Fig. 5a-d).

The scenarios modeled in Fig. $5 \mathrm{a}$ and $5 \mathrm{c}, 5 \mathrm{~b}$ and $5 \mathrm{~d}, 5 \mathrm{e}$ and $5 \mathrm{~g}, 5 \mathrm{f}$ and $5 \mathrm{~h}$ differ only by the propagation direction of the excitation, with atmospheric conditions being the same for the two figures in each pair. The first figure in each pair represents buoyancy resonances for eastward and the second figure-for westward propagation of the excitation. The figures show that reversal of the propagation direction has as strong an effect on the buoyancy resonances as diurnal variations, if not stronger (compare Fig. 5a with Fig. 5b and c). The strong dependence on the propagation direction demonstrates, again, the significance of proper modeling of wind for prediction and understanding of the buoyancy resonances.
Additional physical characteristics of the resonances occurring under atmospheric conditions and excitation conditions explored in Fig. 5a, b are shown in Figs. 7a-c and $7 \mathrm{~d}-\mathrm{e}$, respectively. These characteristics have been calculated in the ray approximation (Georges 1972; Gossard and Hooke 1975; Godin 2015) using the AGW dispersion relation Eq. (5) and the asymptotic resonance condition Eq. (10). Wave dissipation (Fig. 7a, d) tends to increase with the resonance order and can reach extremely high values for higher order resonances. This can be understood, qualitatively, as resulting from the higher order resonances having shorter vertical wavelengths than low-order resonances. Figure $7 \mathrm{a}, \mathrm{d}$ illustrates how imposing the limit of $1 \mathrm{~dB}$ on the absorption restricts the well-defined resonances shown in Fig. 5a, b to $n \leq 15$. Dependence of the absorption on frequency is more complicated than on $n$. For a given resonance order, the change in resonance frequency is accompanied by changes in the trace velocity $V$ and the turning point altitude (Fig. 5). Under atmospheric conditions of Fig. 5a, various effects nearly cancel each other, leaving only a relatively weak trend of absorption increasing at the resonance frequencies approaching their upper limit (Fig. 7a). Under atmospheric conditions of Fig. 5b, this trend is complemented by a much faster absorption increase at the resonance frequencies approaching their lower limit
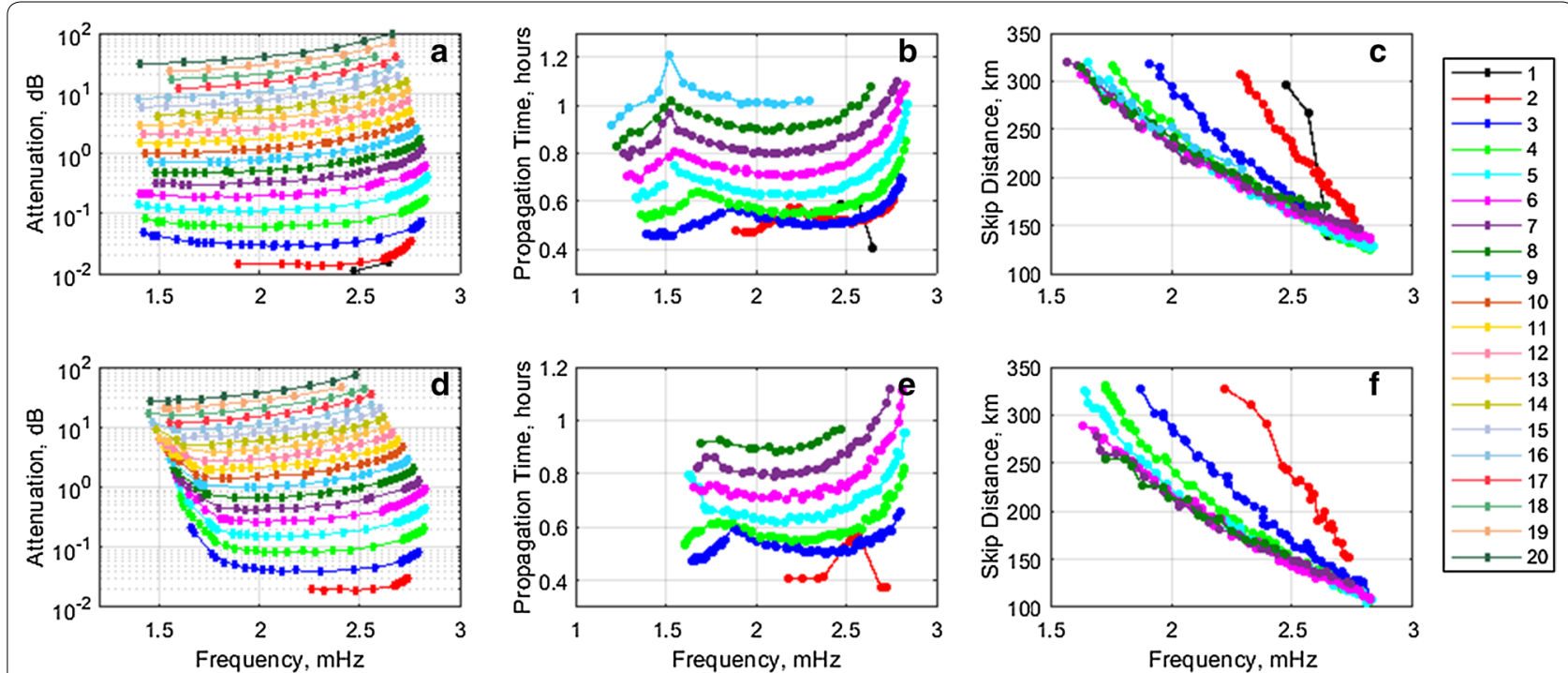

Fig. 7 Properties of buoyancy resonances in a mid-latitude atmosphere. AGW attenuation, travel time, and distance between consecutive returns to the ground level (skip distance) are calculated asymptotically in the ray approximation (Georges 1972; Gossard and Hooke 1975; Godin 2015) for a discrete subset of resonance frequencies under the atmospheric conditions at Wallops Island, Virginia, at GMT 06:00 (a-c) and GMT 18:00 (d-e) on 15 January 2014. The background atmospheric temperature and wind velocity profiles used in the simulations are obtained with the NRLMSISE-00.2 (Picone et al. 2002) and HWM14 (Drob et al. 2015) models, respectively. Different orders $n$ of the resonances are distinguished by color of symbols and indicated in the figures. $\mathbf{a}, \mathbf{d}$ Wave attenuation due to air viscosity and thermal conductivity (Godin 2014) on the path from the ground level to the turning point is shown in $\mathrm{dB}$ for resonances with $n \leq 20$. $\mathbf{b}$, e AGW propagation time from the ground level to the turning point is shown for the well-defined resonances with the attenuation not exceeding $1 \mathrm{~dB}$. $\mathbf{c}$, e Same as in $\mathbf{b}$, e but for the skip distance of AGW rays corresponding to the resonances 
(Fig. 7b), which limits the frequency range where the resonances with $n=6-8$ are well-defined.

Atmospheric resonances have been modeled assuming time-independent environmental conditions. Temporal variability of the atmosphere limits the physical significance of the predicted buoyancy resonances. For a particular resonance to be observable, its frequency should be large compared to the reciprocal of the time $T_{\mathrm{var}}$ it takes for the frequency to change appreciably. Moreover, for individual resonances to be resolvable, the difference between resonance frequencies of consecutive resonances (at a given trace velocity) should be also large compared to $1 / T_{\text {var }}$ In the Wallops Island examples considered above, with a possible exception for the fundamental $(n=1)$ buoyancy resonance, the resonances shown in Fig. 5 have periods less than 17 min, which is small compared to the representative timescale of the resonance frequency variation. The round-trip time between the ground level to the turning point at $z=z_{t}$, which equals twice the AGW propagation between altitudes $z=0$ and $z=z_{t}$ and can be viewed as the minimum time necessary for a resonance to manifest itself, is also within several hours (Fig. 7b, e). Hence, the assumption of time-independent atmospheric conditions appears to be reasonable for prediction of the buoyancy resonances of temperate atmosphere. On the other hand, frequencies of certain buoyancy resonances, especially at smaller trace velocities of tens of $\mathrm{m} / \mathrm{s}$, may become separated by less than $0.1 \mathrm{mHz}$ (Fig. 5), which is too close for these resonances to be individually resolved over the period of observation of several hours, or about $10^{4} \mathrm{~s}$, when variations of the resonance frequencies remain relatively small.

Waves travel along the rays that correspond to the resonances, from the ground level upward to the turning point, then from the turning point back to the ground level, are reflected there, and the process repeats. The rays are spatially periodic curves, and the length of the spatial period, which is usually referred to as the skip distance or the ray cycle length, can be calculated as the distance between two consecutive reflection points at $z=0$ [see, e.g., Chap. 5 in Brekhovskikh and Godin (1999)]. Our asymptotic theory and numerical models assume horizontally invariant atmosphere. For the results to be applicable to real atmosphere, the skip distance should be small compared to the representative spatial scale of environmental variations in the horizontal directions [see, e.g., Chap. 7 in Brekhovskikh and Godin (1999)]. In the Wallops Island examples considered above, the skip distances vary between about 100-350 km (Fig. 7c, f). Away from mountain ranges and similarly strong topographic features, the assumption of the background atmosphere being horizontally invariant within a few degrees in longitude and latitude appears reasonable for prediction of the atmospheric resonances.

\section{Coupling to infragravity waves in the ocean}

Recent theoretical analyses and comparison with observations (Godin et al. 2015; Zabotin et al. 2016) indicated that infragravity waves in the ocean can be major sources of wave activity in the middle and upper atmosphere. Analyses in (Godin et al. 2015; Zabotin et al. 2016) assumed simplified atmospheric models, where AGWs radiated at the ocean surface level travel upwards through the atmosphere without any appreciable reflection. Here we discuss qualitatively the implications that properties of the atmospheric resonances, which are described above, have on this mechanism of excitation of the background atmospheric wave activity.

When atmospheric resonances are excited, the amplitude of atmospheric oscillations increases in a wide range of altitudes (Figs. 1b, 3b, and 4). Efficient excitation of resonances of the atmosphere by waves propagating along its lower boundary requires that, for the trace velocity of the wave, the frequencies of the excitation and a resonance coincide. Velocities of the infragravity waves are less than the sound speed in air (Webb et al. 1991; Herbers et al. 1995; Godin et al. 2015). Hence, only buoyancy resonances can significantly affect AGW generation.

In the open ocean, phase speed of infragravity waves (IGWs), which are surface waves with periods longer than $30 \mathrm{~s}$ (Webb et al. 1991; Herbers et al. 1995; Godin et al. 2014), is a continuous function of their frequency. IGW phase speed steadily increases with water depth and decreases with frequency. For waves in the atmosphere above the ocean, the IGW phase speed serves as the trace speed, and the AGW-IGW coupling is strongly affected by bathymetry. Distribution of water depths in the northwest Atlantic is bimodal (Fig. 8a, b) with the two highest peaks of the probability density function corresponding to the coastal ocean and the open ocean. IGW dispersion curves are shown in Fig. $5 \mathrm{a}-\mathrm{h}$ for four water depths. One of these depths, $150 \mathrm{~m}$, is a representative value of the water depth in the coastal areas (see yellow lines in Figs. 5 and 6b). White lines in Fig. 5a-h correspond to the depths of $4300 \mathrm{~m}$ and $5900 \mathrm{~m}$, which bound the range of typical abyssal plain depths. $55 \%$ of the northwest Atlantic seafloor (by area) is within this depth range (Fig. 8b). The fourth IGW dispersion curve (red lines in Fig. 5a-h) is for the depth of $2649 \mathrm{~m}$ at the location of NDBC station 44402, where long time series of IGW measurements with a seafloor pressure sensor are available (Zabotin et al. 2016).

IGWs in the ocean generate AGWs in the atmosphere regardless of existence of atmospheric resonances. However, if buoyancy resonances are present, the amplitude 

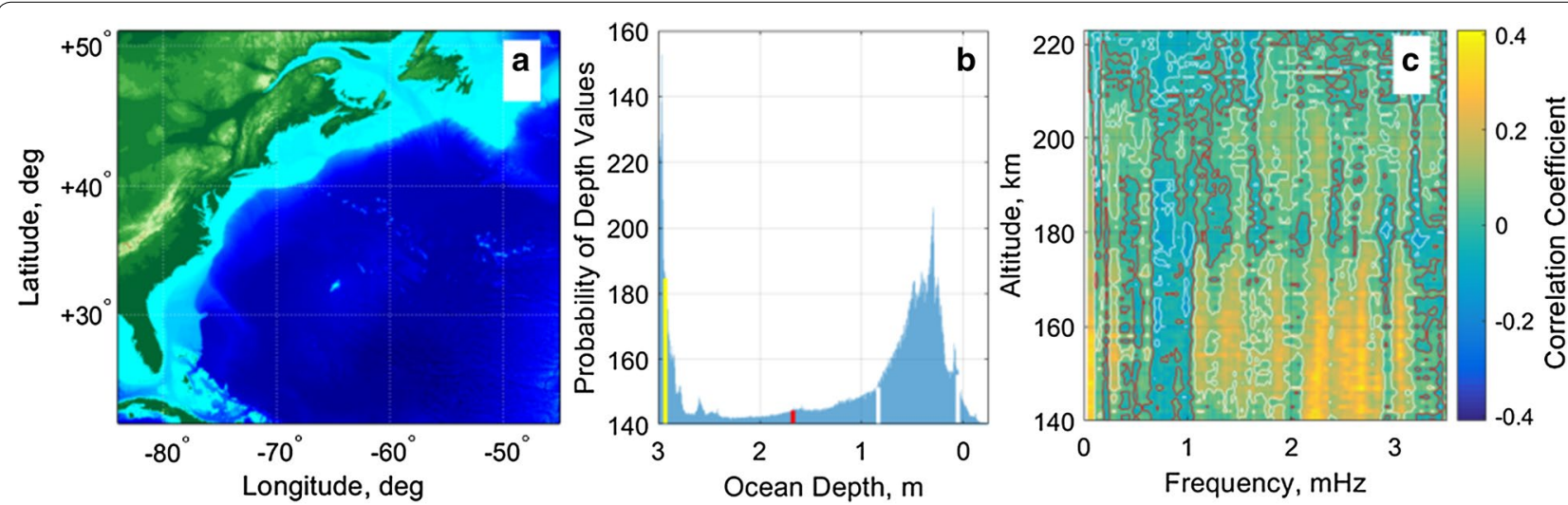

Fig. 8 Bathymetry of northwest Atlantic and correlation of the oceanic and atmospheric wave activity observed in the area. a Bathymetry map of northwest Atlantic. b Probability density function of water depths in the part of the Atlantic Ocean depicted in $\mathbf{a}$. Depth distribution is calculated in the area from $20^{\circ}$ to $50^{\circ} \mathrm{N}$ and from $40^{\circ}$ to $80^{\circ} \mathrm{W}$ using the satellite altimetry-derived global 2-min bathymetry dataset (Smith and Sandwell 1997) provided in MATLAB. The four water depths indicated by color, $150 \mathrm{~m}$ (yellow), $2649 \mathrm{~m}$ (red), $4300 \mathrm{~m}$ and $5900 \mathrm{~m}$ (white), are the depth for which the infragravity wave dispersion curves are shown in Fig. 5. c Correlation coefficient of spectral amplitudes of the wave activities observed in the Atlantic Ocean at the location of NDBC Station $44402\left(39.30^{\circ} \mathrm{N} 70.66^{\circ} \mathrm{W}\right.$ ) and in the thermosphere over the Wallops Island, Virginia (c is adapted from (Zabotin et al. 2016).)

of the atmospheric response increases sharply (Fig. 4) when IGW frequency coincides with a resonance frequency. Using Wallops Island atmospheric conditions as an example, Fig. 5 shows that, in a wide range of water depths, the IGW dispersion curve intersects dispersion curves of several buoyancy resonances of the midlatitude atmosphere. Figure 5 indicates that, for a given direction of IGW propagation, atmospheric oscillations would be preferentially excited by oceanic waves at several discrete frequencies within approximately $1-3 \mathrm{mHz}$ frequency range. However, IGW field in the ocean is usually diffuse, with waves propagating in various horizontal directions, at least when observed over time periods of weeks and months (Godin et al. 2014). For each atmospheric resonance, its frequency at the IGW trace velocity depends on the IGW propagation direction (see Fig. 5). Hence, for diffuse IGWs, the selected frequencies of enhanced (resonance) AGW excitation become selected frequency bands of enhanced excitation.

Comparison of long-term, concurrent observations of thermospheric wave activity with observations of infragravity waves in the ocean show strong correlation of these wave processes (Zabotin et al. 2016). Figure $8 \mathrm{c}$, which is adapted from that reference, illustrates the correlation of the power spectrum density of thermospheric waves, as measured by a Dynasonde radar system located at Wallops Island, with the power spectrum density of IGWs measured at a Deep-ocean Assessment and Reporting of Tsunamis (DART) station 44402 located in northwest Atlantic $415 \mathrm{~km}$ from the Wallops Island at depth of $2649 \mathrm{~m}$. The data used to produce Fig. 8c were collected continuously from 6 May 2013 to
31 January 2014 and analyzed in 12-h sliding windows with 1-h step, resulting in a high statistical significance of the correlation coefficient values shown in the figure (Zabotin et al. 2016). High correlations of spectral densities of mesospheric and oceanic wave activities are expected when oscillations of the ocean surface lead to a strong excitation of atmospheric waves, which propagate to the upper atmosphere. Note several bands of high correlation between $1 \mathrm{mHz}$ and $3.2 \mathrm{mHz}$ in Fig. 8c. Collectively, these bands occupy the range of frequencies where excitation of atmospheric resonances by IGWs is expected (Fig. 5). The band structure itself is also consistent, at least qualitatively, with what is expected from excitation of buoyancy resonances by a diffuse IGW field. Hence, atmospheric resonances may provide a possible explanation of the previously unexplained observations of the band structure of the IGW-AGW correlations. Further research is necessary to determine whether the difference in the altitude extent of the high correlation bands below and above $\sim 1.6 \mathrm{mHz}$ in Fig. $8 \mathrm{c}$ is related to the change in the position of AGW turning point in the buoyancy resonances.

\section{Conclusion}

Resonance oscillations occur in the atmosphere when upward propagating waves are strongly refracted by temperature and wind velocity gradients, reach a turning point at some altitude, and the resulting combination of the upward and downward propagating waves satisfies the boundary conditions at the lower boundary of the atmosphere. Resonances occur on both acoustic and gravity (or buoyancy) branches of the dispersion curve 
of acoustic-gravity waves (AGWs). Accurate prediction of resonance frequencies requires a careful account for the phase changes that AGWs experience at propagation from the ground level to the turning point, at the turning point, and at reflection from the lower boundary. Resonance frequencies are not directly related to the acoustic cutoff frequency or buoyancy frequency at any particular altitude. Atmospheric resonances can be viewed as those normal modes of the coupled atmosphere-ocean-solid earth system that are predominantly concentrated in the atmosphere.

Atmosphere typically supports a few low-order acoustic resonances with periods of $3-5 \mathrm{~min}$ and more numerous lower frequency buoyancy resonances with periods from about $5.7 \mathrm{~min}$ to several hours. Inertial effects due to Earth's rotation significantly affect buoyancy resonances with periods longer than about $4 \mathrm{~h}$. Acoustic resonances are found to be sensitive to the neutral temperature profile in the atmosphere, especially around mesopause and tropopause, and are insensitive to wind. In contrast, buoyancy resonances exhibit high sensitivity to the wind velocity profile and its variations, in addition to the temperature profile sensitivity. Our model predicts significant variations of buoyancy resonances on diurnal timescales, which are primarily caused by wind variations. Individual buoyancy resonances can be resolved and have a direct physical meaning only as long as the resonance frequencies and the difference of frequencies of consecutive resonances are large compared to the inverse of the temporal scale of the resonance frequency variation.

Wave activity in acoustic resonances extends from the ground level to the middle and upper atmosphere. Corresponding AGWs have a mesospheric turning point. The low-temperature layer around the mesopause is a "potential barrier" for the AGWs, where the waves become evanescent. The finite vertical extent of the barrier plays a dual role in the acoustic resonances. On the one hand, the barrier ensures a strong reflection from the mesospheric turning point, which is necessary for a welldefined resonance to occur. On the other hand, the AGW tunneling through the barrier into the thermosphere couples ground-level vibrations with upper atmosphere and underlies most of the observed ionospheric manifestations of the acoustic resonances.

Acoustic resonances are predicted to be a seasonal phenomenon in Antarctic atmosphere. This is traced back to the tropopause temperature dropping below the mesopause temperature in winter.

Theoretical analysis and numerical modeling show that the strongest coupling of the wave activity in the middle and upper atmosphere with waves propagating along the lower boundary of the atmosphere occur when atmospheric resonances are excited. In mid-latitude atmosphere, dispersion curves of several well-defined buoyancy resonances are found to intersect with dispersion curves of infragravity waves (IGWs) in the ocean in a wide range of ocean depths. The exact resonance order and frequency of the intersection depend on the ocean depth and, at a given location, on the direction of IGW propagation. The intersections result in the resonance excitation of atmospheric waves over oceans by IGWs in several frequency bands within approximately $1-3 \mathrm{mHz}$ frequency range. Excitation of atmospheric resonances by IGWs provides a possible explanation of the observations (Zabotin et al. 2016) of the bands of high correlation between spectra of IGWs off the US East Coast and wave activity in the ionosphere above Wallops Island, Virginia.

Waves in the atmosphere are excited by vertical displacements of its lower boundary most efficiently at the resonance frequencies. However, the resonances exist regardless of a particular way of the wave generation. Same resonances will be also excited by sources within the atmosphere, with the efficiency dependent of the source type and altitude. The long vertical wavelength of acoustic resonances (Figs. 1 and 3) indicates that, in agreement with observations (Bedard 1978; Jones and Georges 1976; Nishioka et al. 2013; Pilger et al. 2013), these resonances will be excited by sources in the troposphere nearly as efficiently as by sources at the ground level.

\section{Abbreviations \\ AGW: Acoustic-gravity wave; IGW: Infragravity wave; WKB: \\ Wentzel-Kramers-Brillouin.}

\section{Acknowledgements}

Helpful discussions with Elisabeth Blanc (CEA, France) are gratefully acknowledged.

\section{Authors' contributions}

OG and NZ conceived this research. OG developed the analytical theory and drafted the manuscript. NZ and LZ performed numerical modeling and computer simulations. All authors read and approved the final manuscript.

\section{Funding}

The research reported in this paper was supported by the National Science Foundation, award OPP-164319, and utilized the research computing facility at the University of Colorado Boulder, which is supported by the National Science Foundation, award CNS-0821794.

\section{Availability of data and materials}

The background air temperature and wind velocity profiles, which have been used in this study in modeling wave fields in the atmosphere, were generated by the atmospheric models NRLMSISE-00.2 (Picone et al. 2002) and HWM14 (Drob et al. 2015). The other data supporting the analysis and conclusions presented above are available in Godin and Zabotin (2016) and Zabotin et al. (2016).

Competing interests

The authors declare that they have no competing interests. 


\section{Author details}

${ }^{1}$ Department of Physics, Naval Postgraduate School, Monterey, CA 93943, USA. ${ }^{2}$ Department of Electrical, Computer, and Energy Engineering, University of Colorado, Boulder, CO 80309, USA.

Received: 12 May 2020 Accepted: 25 August 2020 Published online: 05 September 2020

\section{References}

Aoyama T, Iyemori T, Nakanishi K, Nishioka M, Rosales D, Veliz O, Safor EV (2016) Localized field-aligned currents and 4-min TEC and ground magnetic oscillations during the 2015 eruption of Chile's Calbuco volcano. Earth Planets Space 68:148. https://doi.org/10.1186/s40623-016-0523-0

Artru J, Farges T, Lognonné P (2004) Acoustic waves generated from seismic surface waves: Propagation properties determined from Doppler sounding observation and normal-mode modeling. Geophys J Int 158:1066-1077

Artru J, Lognonné P, Blanc E (2001) Normal modes modelling of post-seismic ionospheric oscillations. Geophys Res Lett 28:697-700

Bedard AJ (1978) Infrasound originating near mountainous regions in Colorado. J Appl Meteorol 17:1014-1022

Brekhovskikh LM, Godin OA (1999) Acoustics of layered media. 2: point sources and bounded beams, 2nd edn. Springer, Heidelberg, pp 150-191

Budden KG, Smith MS (1976) Phase memory and additional memory in WKB solutions for wave propagation in stratified media. Proc R Soc Lond A 350(1660):27-46

Cahyadi MN, Heki K (2014) Coseismic ionospheric disturbance of the large strike-slip earthquakes in North Sumatra in 2012: $\mathrm{M}_{\mathrm{w}}$ dependence of the disturbance amplitudes. Geophys J Int 200:116-129

Chen C, Chu X (2017) Two-dimensional Morlet wavelet transform and its application to wave recognition methodology of automatically extracting two-dimensional wave packets from lidar observations in Antarctica. J Atm Solar-Terrestrial Phys 162:28-47

Chen C, Chu X, Zhao J, Roberts BR, Yu Z, Fong W, Lu X, Smith JA (2016) Lidar observations of persistent gravity waves with periods of 3-10 $\mathrm{h}$ in the Antarctic middle and upper atmosphere at McMurdo $\left(77.83^{\circ} \mathrm{S}, 166.67^{\circ} \mathrm{E}\right)$. J Geophys Res Space Phys 121:1483-1502

Chimonas G, Peltier WR (1974) On severe storm acoustic signals observed at ionospheric heights. J Atm Solar-Terrestrial Phys 36:821-828

Choosakul N, Saito A, lyemori T, Hashizume M (2009) Excitation of 4-min periodic ionospheric variations following the great Sumatra-Andaman earthquake in 2004. J Geophys Res Space Phys 114:A10313. https://doi. org/10.1029/2008JA013915

Drob DP, Emmert JT, Meriwether JW, Makela JJ, Doornbos E, Conde M, Hernandez G, Noto J, Zawdie KA, McDonald SE, Huba JD (2015) An update to the Horizontal Wind Model (HWM): the quiet time thermosphere. Earth Space Sci 2:301-319

Einaudi F, Hines CO (1970) WKB approximation in application to acousticgravity waves. Can J Phys 48:1458-1471

Fritts DC, Alexander MJ (2003) Gravity wave dynamics and effects in the middle atmosphere. Rev Geophys 41:1003

Georges TM (1972) 3D ray tracing for acoustic-gravity waves. In: Effects of atmospheric acoustic gravity waves on electromagnetic wave propagation, AGARD conference proceedings 115: 2-1-2-8. Hatford House, London

Gill AE (1982) Atmosphere-ocean dynamics. Academic, New York

Godin OA (1997) Reciprocity and energy theorems for waves in a compressible inhomogeneous moving fluid. Wave Motion 25:143-167. https://doi. org/10.1016/S0165-2125(96)00037-6

Godin OA (2014) Dissipation of acoustic-gravity waves: an asymptotic approach. J Acoust Soc Am 136:EL411-EL417

Godin OA (2015) Wentzel-Kramers-Brillouin approximation for atmospheric waves. J Fluid Mech 777:260-290

Godin OA (2016) Diffraction of acoustic-gravity waves in the presence of a turning point. J Acoust Soc Am 140:283-295

Godin OA, Naugolnykh KA (2005) Guided propagation of naturally occurring infrasound in troposphere and stratosphere. Geophys Res Lett 32:3824
Godin OA, Zabotin NA (2016) Resonance vibrations of the Ross Ice Shelf and observations of persistent atmospheric waves. J Geophys Res Space Phys 121:10157-10171

Godin OA, Zabotin NA, Sheehan AF, Collins JA (2014) Interferometry of infragravity waves off New Zealand. J Geophys Res Oceans 118:1103-1122. https://doi.org/10.1002/2013JC009395

Godin OA, Zabotin NA, Bullett TW (2015) Acoustic-gravity waves in the atmosphere generated by infragravity waves in the ocean. Earth Planets Space 67:47. https://doi.org/10.1186/s40623-015-0212-4

Golitsyn GS (1965) Damping of small oscillations in the atmosphere due to viscosity and thermal conductivity. Izv Acad Sci USSR Atmos Oceanic Phys 1:82-89

Gossard EE, Hooke WH (1975) Waves in the atmosphere. Elsevier, Amsterdam Heidarzadeh M, Satake K (2013) Waveform and spectral analyses of the 2011 Japan tsunami records on tide gauge and DART stations across the Pacific Ocean. Pure Appl Geophys 170:1275-1293

Herbers THC, Elgar S, Guza RT (1995) Generation and propagation of infragravity waves. J Geophys Res 100:24863-24872. https://doi. org/10.1029/95JC02680

Hines CO (1965) Atmospheric gravity waves-a new toy for the wave theorist. J Res NBS Radio Sci 69D:375-380

Iyemori T, Tanaka Y, Odagi Y, Sano Y, Takeda M, Nose M, Utsugi M, Rosales D, Choque E, Ishitsuka J, Yamanaka S (2013) Barometric and magnetic observations of vertical acoustic resonance and resultant generation of field-aligned current associated with earthquakes. Earth Planets Space 65:901-909. https://doi.org/10.5047/eps.2013.02.002

Jin S (2018) Two-mode ionospheric disturbances following the 2005 Northern California offshore earthquake from GPS measurements. J Geophys Res Space Phys 123:8587-8598

Jones M, Georges TM (1976) Infrasound from convective storms. III. Propagation to the ionosphere. J Acoust Soc Am 59:765-779

Kanamori RH, Mori J, Harkrider DG (1994) Excitation of atmospheric oscillations by volcanic eruptions. J Geophys Res Solid Earth 99:21947-21961

Kobayashi N (2007) A new method to calculate normal modes. Geophys J Int 168:315-331

Liu Y, Jin S (2019) Ionospheric Rayleigh wave disturbances following the 2018 Alaska earthquake from GPS observations. Remote Sensing 11:901. https ://doi.org/10.3390/rs11080901

Lognonné P, Clévédé E, Kanamori H (1998) Computation of seismograms and atmospheric oscillations by normal-mode summation for a spherical earth model with realistic atmosphere. Geophys J Int 135:388-406

Matsumura M, Iyenori T, Tanaka Y, Han D, Nose M, Utsugi M, Oshiman N, Shinagawa H, Odagi Y, Tabata Y (2009) Acoustic resonance between ground and thermosphere. Data Sci J 8:S68-S77

Matsumura M, Saito A, lyenori T, Shinagawa H, Tsugawa T, Otsuka Y, Nishioka M, Chen CH (2011) Numerical simulations of atmospheric waves excited by the 2011 off the Pacific coast of Tohoku earthquake. Earth Planets Space 63:885-889. https://doi.org/10.5047/eps.2011.07.015

Matsumura M, Shinagawa H, lyemori T (2012) Horizontal extension of acoustic resonance between the ground and the lower thermosphere. J Atm Solar-Terrestrial Phys 75:127-132

Nakashima Y, Heki K, Takeo A, Cahyadi MN, Aditiya A, Yoshizawa K (2016) Atmospheric resonant oscillations by the 2014 eruption of the Kelud volcano, Indonesia, observed with the ionospheric total electron contents and seismic signals. Earth Planet Sci Lett 434:112-116

Nishida K (2014) Source spectra of seismic hum. Geophys J Int 199:416-429

Nishioka M, Tsugawa T, Kubota M, Ishii M (2013) Concentric waves and shortperiod oscillations observed in the ionosphere after the 2013 Moore EF5 tornado. Geophys Res Lett 40:5581-5586

Ogawa T, Nishitani N, Tsugawa T, Shiokawa K (2012) Giant ionospheric disturbances observed with the SuperDARN Hokkaido HF radar and GPS network after the 2011 Tohoku earthquake. Earth Planets Space 64:1295-1307. https://doi.org/10.5047/eps.2012.08.001

Ozawa S, Nishimura T, Suito H, Kobayashi T, Tobita M, Imakiire T (2011) Coseismic and postseismic slip of the 2011 magnitude-9 Tohoku-Oki earthquake. Nature 475(7356):373-376. https://doi.org/10.1038/nature10227

Picone JM, Hedin AE, Drob DP, Aikin AC (2002) NRLMSISE-00 empirical model of the atmosphere: statistical comparisons and scientific issues. J Geophys Res Space Phys 107:1468. https://doi.org/10.1029/2002JA009430 
Pilger C, Schmidt C, Bittner M (2013) Statistical analysis of infrasound signatures in airglow observations: indications for acoustic resonance. J Atm Solar-Terrestrial Phys 93:70-79

Pitteway MLV, Hines CO (1965) The reflection and ducting of atmospheric acoustic-gravity waves. Can J Phys 43:2222-2243

Reddy CD, Shrivastava MN, Seemala GK, González G, Baez JC (2016) lonospheric plasma response to Mw 8.3 Chile Illapel earthquake on September 16, 2015. Pure Appl Geophys 173:1451-1461

Rolland LM, Lognonné P, Astafyeva E, Kherani EA, Kobayashi N, Mann M, Munekane $\mathrm{H}$ (2011) The resonant response of the ionosphere imaged after the 2011 off the Pacific coast of Tohoku Earthquake. Earth Planets Space 63:853-857. https://doi.org/10.5047/eps.2011.06.020

Saito A, Tsugawa T, Otsuka Y, Nishioka M, Iyemori T, Matsumura M, Saito S, Chen CH, Goi Y, Choosakul N (2011) Acoustic resonance and plasma depletion detected by GPS total electron content observation after the 2011 off the Pacific coast of Tohoku Earthquake. Earth Planets Space 63:863-867. https://doi.org/10.5047/eps.2011.06.034

Shinagawa H, Iyemori T, Saito S, Maruyama T (2007) A numerical simulation of ionospheric and atmospheric variations associated with the Sumatra earthquake on December 26, 2004. Earth Planets Space 59:1015-1026. https://doi.org/10.1186/BF03352042

Smith WHF, Sandwell DT (1997) Global seafloor topography from satellite altimetry and ship depth soundings. Science 277:1957-1962. https://doi. org/10.1126/science.277.5334.1956

Tahira M (1995) Acoustic resonance of the atmosphere at 3.7 Hz. J Atmos Sci 52:2670-2674
Taroyan Y, Erdélyi R (2008) Global acoustic resonance in a stratified solar atmosphere. Solar Phys 251:523-531

Watada S, Kanamori H (2010) Acoustic resonant oscillations between the atmosphere and the solid earth during the 1991 Mt. Pinatubo eruption. J Geophys Res Solid Earth 115:2319

Webb SC, Zhang X, Crawford W (1991) Infragravity waves in the deep ocean. J Geophys Res 96:2723-2736. https://doi.org/10.1029/90JC02212

Widmer R, Zürn W (1992) Bichromatic excitation of long-period Rayleigh and air waves by the Mount Pinatubo and El Chichón volcanic eruptions. Geophys Res Lett 19:765-768

Yuan D, Su J, Jiao F, Walsh RW (2016) Stochastic transients as a source of quasiperiodic processes in the solar atmosphere. Astrophys J Suppl Ser 224:30

Zabotin NA, Godin OA, Bullett TW (2016) Ocean is a major source of waves in the thermosphere. J Geophys Res Space Phys 121:3452-3463. https://doi. org/10.1002/2016JA022357

Zürn W, Widmer R (1996) Worldwide observation of bichromatic long-period Rayleigh-waves excited during the June 15, 1991 Eruption of Mt. Pinatubo. In: Newhall C, Punongbayan JR (eds) Fire and Mud, Eruptions of Mount Pinatubo, Philippines. Quezo City and University of Washington Press, Philippine Institute of Volcanology and Seismology, pp 615-624

\section{Publisher's Note}

Springer Nature remains neutral with regard to jurisdictional claims in published maps and institutional affiliations.

\section{Submit your manuscript to a SpringerOpen ${ }^{\circ}$ journal and benefit from:}

- Convenient online submission

- Rigorous peer review

- Open access: articles freely available online

- High visibility within the field

- Retaining the copyright to your article

Submit your next manuscript at springeropen.com 